NASA Contractor Report 187462

ICASE Report 90-79

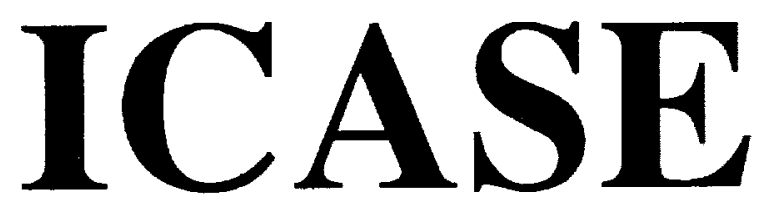

\title{
A FULLY SINC-GALERKIN METHOD FOR EULER-BERNOULLI BEAM MODELS
}

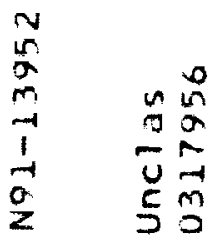
R. C. Smith
K. L. Bowers
J. Lund

Contract No. NAS1-18605

October 1990

Institute for Computer Applications in Science and Engineering NASA Langley Research Center

Hampton, Virginia 23665-5225

Operated by the Universities Space Research Association

\section{N/S/ \\ Nalional Aeronaulics and \\ Space Adinimistration}

$$
\sum_{0}^{5}
$$

Langley Research Center

Hamplon, Virginia 23665-5225 


\title{
A FULLY SINC-GALERKIN METHOD FOR EULER-BERNOULLI BEAM MODELS
}

\author{
R.C. Smith ${ }^{1}$ \\ Institute for Computer Applications in Science and Engineering \\ NASA Langley Research Center \\ Hampton, VA 23665 \\ K.L. Bowers and J. Lund \\ Department of Mathematical Sciences \\ Montana State University \\ Bozeman, MT 59717
}

\begin{abstract}
A fully Sinc-Galerkin method in both space and time is presented for fourth-order timedependent partial differential equations with fixed and cantilever boundary conditions. The sinc discretizations for the second-order temporal problem and the fourth-order spatial problems are presented. Alternate formulations for variable parameter fourth-order problems are given which prove to be especially useful when applying the forward techniques of this paper to parameter recovery problems. The discrete system which corresponds to the timedependent partial differential equations of interest are then formulated. Computational issues are discussed and a robust and efficient algorithm for solving the resulting matrix system is outlined. Numerical results which highlight the method are given for problems with both analytic and singular solutions as well as fixed and cantilever boundary conditions.
\end{abstract}

\footnotetext{
${ }^{1}$ This research was supported by the National Aeronautics and Space Administration under NASA Contract No. NAS1-18605 while the author was in residence at the Institute for Computer Applications in Science and Engineering (ICASE), NASA Langley Research Center, Hampton, VA 23665.
} 


\section{Introduction}

The Sinc-Galerkin method for partial differential equations (PDE's) has previously been developed for the model elliptic problem in two and three dimensions $[1,2]$, the parabolic problem in one and two dimensions [3,2], and the second-order hyperbolic problem in one dimension [4]. The present work extends the method to fourth-order time-dependent problems with various common boundary conditions. This extension is important for the very practical reason that the numerical solution of problems in this class is necessary in applications ranging from the control of large flexible space structures to the development of robotics designs $[5,6,7]$.

For clarity of development, the method will be presented for the linear fourth-order timedependent problems

$$
\begin{gathered}
\mathcal{L} u(x, t) \equiv \frac{\partial^{2} u}{\partial t^{2}}(x, t)+\frac{\partial^{2}}{\partial x^{2}}\left(E I(x) \frac{\partial^{2} u}{\partial x^{2}}(x, t)\right)=f(x, t), \quad 0<x<1 \quad t>0 \\
u(0, t)=u(1, t)=0, \quad t>0 \\
\frac{\partial u}{\partial x}(0, t)=\frac{\partial u}{\partial x}(1, t)=0, \quad t>0 \\
u(x, 0)=\frac{\partial u}{\partial t}(x, 0)=0, \quad 0 \leq x \leq 1
\end{gathered}
$$

and

$$
\begin{gathered}
\mathcal{L} u(x, t)=f(x, t), \quad 0<x<1 \quad t>0 \\
u(0, t)=\bar{\alpha}(t), \quad\left(E I \frac{\partial^{2} u}{\partial x^{2}}\right)(1, t)=\bar{\gamma}(t), \quad t>0 \\
\frac{\partial u}{\partial x}(0, t)=\bar{\beta}(t), \quad \frac{\partial}{\partial x}\left(E I \frac{\partial^{2} u}{\partial x^{2}}\right)(1, t)=\bar{\delta}(t), \quad t>0 \\
u(x, 0)=\frac{\partial u}{\partial t}(x, 0)=0, \quad 0 \leq x \leq 1 .
\end{gathered}
$$

These formulations are generalizations of the equations which arise when using the EulerBernoulli theory to model beams with flexural rigidity $E I(x)$ and fixed and cantilevered ends, respectively. The general $\bar{\gamma}(t)$ and $\bar{\delta}(t)$ in (1.2) allow for the inclusion of boundary controllers. For ease of presentation, the boundary conditions in (1.1) and (1.2) will respectively be referred to as fixed and cantilever conditions throughout the paper. It is noted that 
the methods of this work are easily extended to problems with simple and free boundary conditions with further details given in [8].

The construction of an approximate solution to problems of the form (1.1) or (1.2) commonly begins with a Galerkin discretization of the spatial variable with time-dependent coefficients. This yields a system of ordinary differential equations which is solved via differencing techinques. Due to stability constraints on the discrete evolution operator, low order methods with small time steps are often required to obtain accurate approximations. In contrast, the method of this work implements a Galerkin scheme in time as well as space. Because the basis functions are tensor products of sinc functions composed with suitable conformal maps, the method has the inherent advantage that the study of error analysis and matrix structure begins at the level of an ordinary differential equation.

The fully Sinc-Galerkin method in space and time has many other salient features due both to the properties of the basis functions and to the manner in which the problem is discretized. First, the judicious choice of a conformal map provides approximate solutions to (1.1) and (1.2) which are valid on the infinite time interval rather than only on a truncated time domain. Furthermore, the optimal exponential convergence rate is maintained even in the presence of boundary singularities. Finally, the discrete system requires no numerical integrations to fill either the coefficient matrix or the right-hand side vector. All three features prove to be advantageous when solving both forward and inverse fourth-order problems. A drawback to the method is that it produces a full system in contrast to the banded matrices which are associated with finite difference and finite element methods. In part, the exponential convergence rate offsets this disadvantage.

The foundations of the Sinc-Galerkin method are described in Section 2. The fundamental quadrature rule is given, and the exponential convergence rate of this method is stated. A thorough review of sinc function properties can be found in [9] and [10].

In the next section, the discretization of the second-order temporal and fourth-order spatial operators is outlined. Two schemes for discretizing $E I(x)$ are presented. These two schemes are motivated on the one hand by the forward problem and on the other hand by the inverse problem involving the recovery of $E I(x)$, given sampled data. In the first, $E I(x)$ is differentiated directly, whereas in the second (as motivated by the parameter recovery 
problem) $E I(x)$ is replaced by a finite dimensional expansion $E I_{m_{*}}$ before differentiation. Attention is focussed on preserving the method's exponential convergence rate while discretizing $E I(x)$ and adapting to varying boundary conditions.

In Section 4, the one-dimensional results from the previous section are combined to yield methods which very accurately approximate the solutions to the fourth-order time-dependent problems (1.1) and (1.2). Two equivalent formulations for the resulting discrete system are presented and a very robust and accurate solution algorithm is outlined.

Numerical results are presented in the fifth section. Of the many examples tested, those discussed in this section best exhibit the features necessary for the practical implementation of the Sinc-Galerkin method. The first and second examples illustrate the method as applied to problems with fixed boundary conditons while the third and fourth examples have cantilever boundary conditions. The first and last examples have analytic solutions, the second example has an algebraic singularity, and the third example contains a logarithmic singularity. The numerical results demonstrate that the exponential convergence rate is maintained in all four cases.

\section{Sinc Function Properties}

For the Sinc-Galerkin method, the basis functions are derived from the Whittaker cardinal (sinc) function

$$
\operatorname{sinc}(x) \equiv \frac{\sin (\pi x)}{\pi x}, \quad-\infty<x<\infty
$$

and its translates

$$
S(k, h)(x) \equiv \operatorname{sinc}\left(\frac{x-k h}{h}\right), \quad h>0 .
$$

For $h^{*}=\frac{\pi}{4}$, three adjacent members of this sinc family $\left(S\left(k, h^{*}\right)(x), k=-1,0,1\right)$ are shown in Figure 1. 


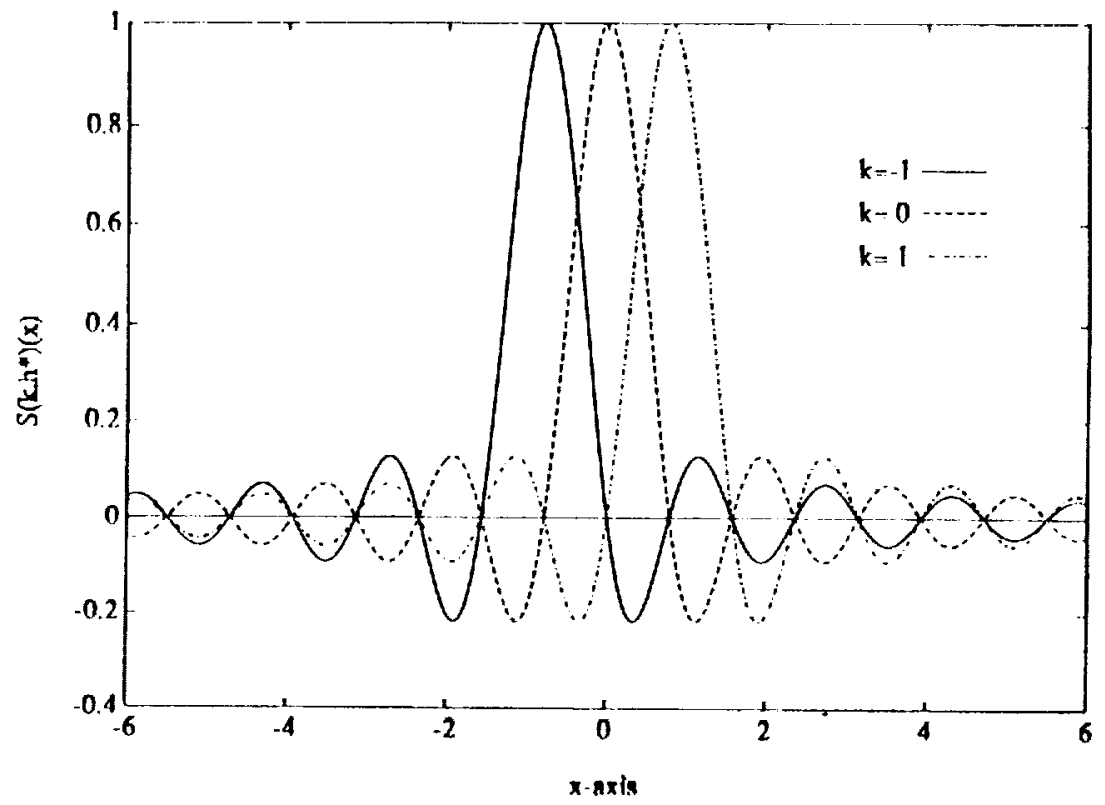

Figure 1. Three Adjacent Members $\left(S\left(k, h^{*}\right)(x), k=-1,0,1, h^{*}=\frac{\pi}{4}\right)$ of the Translated Sinc Family.

To construct basis functions on the intervals $(0,1)$ and $(0, \infty)$, respectively, consider the conformal maps

$$
\phi(z)=\ln \left(\frac{z}{1-z}\right)
$$

and

$$
\Upsilon(w)=\ln (w)
$$

The map $\phi$ carries the eye-shaped region

$$
D_{E}=\left\{z=x+i y:\left|\arg \left(\frac{z}{1-z}\right)\right|<d \leq \frac{\pi}{2}\right\}
$$

onto the infinite strip

$$
D_{s}=\left\{\xi=\zeta+i \eta:|\eta|<d \leq \frac{\pi}{2}\right\}
$$

Similarly, the map $\Upsilon$ carries the infinite wedge

$$
D_{W}=\left\{w=t+i s:|\arg (w)|<d \leq \frac{\pi}{2}\right\}
$$

onto the strip $D_{s}$. These regions are depicted in Figure 2. 

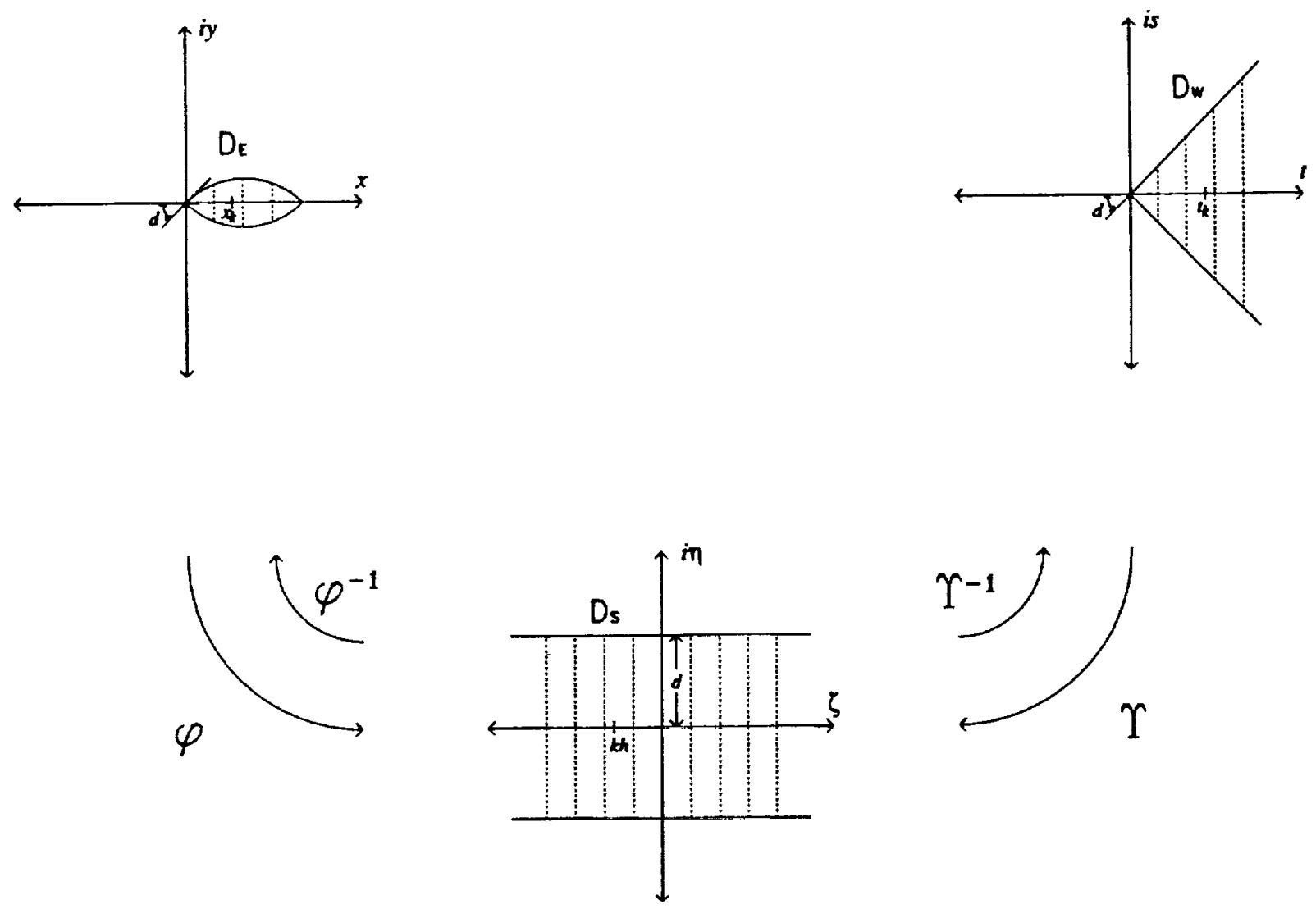

Figure 2. The Domains $D_{S}, D_{E}$, and $D_{W}$.

The sinc gridpoints $z_{k} \in(0,1)$ in $D_{E}$ will be denoted $x_{k}$ since they are real. Similarly, the gridpoints $w_{k} \in(0, \infty)$ in $D_{w}$ will be denoted $t_{k}$. Both are inverse images of the equispaced grid in $D_{S}$; that is

$$
x_{k}=\phi^{-1}(k h)=\frac{e^{k h}}{1+e^{k h}}
$$

and

$$
t_{k}=\Upsilon^{-1}(k h)=e^{k h}
$$

To simplify notation throughout the remainder of this section, the pairs $\phi, D_{E}$ and $\Upsilon, D_{W}$ are referred to generically as $\chi, D$. It is understood that the subsequent definition and theorems hold in either setting. Furthermore, the inverse of $\chi$ is denoted by $\psi$.

The important class of functions for sinc interpolation and quadrature is denoted $B(D)$ and defined next. 
Definition 2.1. Let $B(D)$ be the class of functions $F$ which are analytic in $D$, satisfy

$$
\int_{\psi(t+L)}|F(z) d z| \rightarrow 0, \quad t \rightarrow \pm \infty
$$

where $L=\left\{i s:|s|<d<\frac{\pi}{2}\right\}$, and on the boundary of $D$ (denoted $\partial D$ ) satisfy

$$
N(F) \equiv \int_{\partial D}|F(z) d z|<\infty .
$$

The following theorem for functions in $B(D)$ is found in [11].

Theorem 2.1. Let $\Gamma$ be $(0,1)$ or $(0, \infty)$ when $\chi=\phi$ or $\Upsilon$, respectively. If $F \in B(D)$ and $z_{j}=\psi(j h)=\chi^{-1}(j h), j=0, \pm 1, \pm 2, \ldots$, then for $h>0$ sufficiently small

$$
\left|\int_{\Gamma} F(z) d z-h \sum_{j=-\infty}^{\infty} \frac{F\left(z_{j}\right)}{\chi^{\prime}\left(z_{j}\right)}\right| \leq K_{1} e^{-2 \pi d / h}
$$

Theorem 2.1 illustrates the exponential convergence rate which is a trademark of sinc methods. There is a common occasion when it is possible to evaluate the infinite series appearing in (2.3), namely when integrating against $S(k, h) \circ \chi$. In general, however, the series must be truncated. With additional hypotheses, it is proven in [9] and [12] that the truncation need not be at the expense of the exponential convergence.

Theorem 2.2. Assume $F \in B(D)$ and that there exist positive constants $K, \alpha$ and $\beta$ such that

$$
\left|\frac{F(\tau)}{\chi^{\prime}(\tau)}\right| \leq K \begin{cases}\exp (-\alpha|\chi(\tau)|), & \tau \in \psi((-\infty, 0)) \\ \exp (-\beta|\chi(\tau)|), & \tau \in \psi([0, \infty))\end{cases}
$$

Then for $h$ sufficiently small

$$
\left|\int_{\Gamma} F(z) d z-h \sum_{j=-M}^{N} \frac{F\left(z_{j}\right)}{\chi^{\prime}\left(z_{j}\right)}\right| \leq K_{1} e^{-2 \pi d / h}+\frac{K}{\alpha} e^{-\alpha M h}+\frac{K}{\beta} e^{-\beta N h} .
$$

Theorems 2.1 and 2.2 are used to establish a uniform error bound when building an approximate solution to an ordinary differential equation (ODE). It should be noted that 
the nature of the class $B(D)$ guarantees that the exponential convergence rate holds for many differential equations with singular solutions; that is, problems where the solution has an unbounded derivative on the boundary. By applying the scheme to select second- and fourth-order ODE's, one can derive the fundamental matrices comprising the discrete sinc system for the fourth-order time-dependent problems of interest.

\section{Sinc-Galerkin Systems for ODE's}

In this section, the sinc discretizations will be catalogued for the second-order temporal problem and two different fourth-order spatial problems distinguished by their boundary conditions. Alternate formulations for the variable-parameter fourth-order problems will also be given which prove to be especially useful when applying the forward techniques outlined in this paper to parameter recovery problems.

In order to construct the discrete Sinc-Galerkin system for either the temporal or spatial problems, the following identities are needed. Let

$$
\begin{gathered}
\left.\delta_{p i}^{(0)} \equiv[S(p, h) \circ \chi(z)]\right|_{z=z_{i}}= \begin{cases}1, & i=p \\
0, & i \neq p,\end{cases} \\
\left.\delta_{p i}^{(1)} \equiv h\left[\frac{d}{d \chi} S(p, h) \circ \chi(z)\right]\right|_{z=z_{i}}= \begin{cases}0, & i=p \\
\frac{(-1)^{i-p}}{(i-p)}, & i \neq p,\end{cases} \\
\left.\delta_{p i}^{(2)} \equiv h^{2}\left[\frac{d^{2}}{d \chi^{2}} S(p, h) \circ \chi(z)\right]\right|_{z=z_{i}}= \begin{cases}\frac{\pi^{2}}{3}, & i=p \\
\frac{(-2)(-1)^{i-p}}{(i-p)^{2}}, & i \neq p,\end{cases} \\
\left.\delta_{p i}^{(3)} \equiv h^{3}\left[\frac{d^{3}}{d \chi^{3}} S(p, h) \circ \chi(z)\right]\right|_{z=z_{i}}= \begin{cases}0, & i=p \\
\frac{(-1)^{i-p}}{(i-p)^{3}}\left[6-\pi^{2}(i-p)^{2}\right], & i \neq p,\end{cases}
\end{gathered}
$$


and

$$
\left.\delta_{p i}^{(4)} \equiv h^{4}\left[\frac{d^{4}}{d \chi^{4}} S(p, h) \circ \chi(z)\right]\right|_{z=x_{i}}= \begin{cases}\frac{\pi^{4}}{5}, & i=p \\ \frac{-4(-1)^{i-p}}{(i-p)^{4}}\left[6-\pi^{2}(i-p)^{2}\right], & i \neq p\end{cases}
$$

denote the evaluation at the gridpoint $z_{i}$ of the sinc-map compositions and their derivatives with respect to the map $\chi$.

\subsection{The Spatial Problem: Fixed Boundary Conditions}

In [13], a thorough analysis of the Sinc-Galerkin method is given for linear fourth-order ODE's with fixed boundary conditions. For purposes of constructing the sinc discretization for (1.1), it suffices to review that procedure for

$$
\begin{gathered}
L u(x) \equiv\left(E I(x) u^{\prime \prime}(x)\right)^{\prime \prime}=f(x), \quad 0<x<1 \\
u(0)=u(1)=0 \\
u^{\prime}(0)=u^{\prime}(1)=0 .
\end{gathered}
$$

Note that the interval $(0,1)$ is for convenience only; adapting the map $\phi$ (see (2.1)) generalizes the method to any finite interval $(a, b)$.

To define the Sinc-Galerkin approximation to (3.6), select the basis $\left\{S_{i}\right\}_{i=-M x}^{N_{*}}$ where $S_{i}(x) \equiv S\left(i, h_{x}\right) \circ \phi(x)$ and take the approximate solution to be

$$
u_{m_{\bullet}}(x)=\sum_{i=-M_{\bullet}}^{N_{*}} u_{i} S_{i}(x), \quad m_{x}=M_{x}+N_{x}+1
$$

The unknown coefficients $\left\{u_{i}\right\}$ in (3.7) are determined by orthogonalizing the residual $L u_{m_{\varepsilon}}-f$ with respect to the functions $\left\{S_{p}\right\}_{p=-M_{\infty}}^{N_{*}}$. This yields the discrete system

$$
\left(L u_{m_{\bullet}}-f, S_{p}\right)=0
$$

for $p=-M_{x}, \cdots, N_{x}$. The weighted inner product $(\cdot, \cdot)$ is taken to be

$$
(F, G)=\int_{0}^{1} F(x) G(x) w(x) d x
$$

where

$$
w(x)=\frac{1}{\left(\phi^{\prime}(x)\right)^{\frac{3}{2}}}
$$


For a further discussion concerning the choice of weight, see [13].

Before invoking the quadrature rules, integration by parts is used to transfer the differentiation of $u$ onto $S_{p} w$, thus yielding the system

$$
\int_{0}^{1} u(x)\left[E I(x)\left(S_{p}(x) w(x)\right)^{\prime \prime}\right]^{\prime \prime} d x=\int_{0}^{1} f(x) S_{p}(x) w(x) d x
$$

for $p=-M_{x}, \cdots, N_{x}$. With the weight choice (3.9), the boundary terms

$$
\left.\left\{\left(E I u^{\prime \prime}\right)^{\prime}\left(S_{p} w\right)-\left(E I u^{\prime \prime}\right)\left(S_{p} w\right)^{\prime}+u^{\prime}\left(E I\left(S_{p} w\right)^{\prime}\right)^{\prime}-u\left(E I\left(S_{p} w\right)^{\prime}\right)^{\prime \prime}\right\}(x)\right|_{0} ^{1}
$$

vanish for essentially all problems of interest.

Two approaches are distinguished by the treatment of the first integral in (3.10). In the traditional scheme, $E I(x)$ is differentiated directly and the resulting integrals are approximated via sinc quadrature rules. This scheme is direct and suitable for a large class of forward problems. The second, alternative, approach is motivated by the parameter recovery problem and differs from the first in that $E I(x)$ is replaced by a sinc expansion, $E I_{m_{\bullet}}$, before quadrature is applied. Both approaches then proceed in the same manner whereby the system is expanded and the resulting integrals are evaluated via Theorem 2.2 , or when possible, Theorem 2.1 .

The careful choice of the decay parameters $\alpha$ and $\beta$ in (2.4) provides a means of balancing the asymptotic errors resulting from the quadrature and hence minimizes the system size. With regard to (2.4), the condition

$$
\left|E I(x) u(x) w(x)\left(\phi^{\prime}(x)\right)^{3}\right| \leq \hat{K} \begin{cases}x^{\alpha}, & x \in\left(0, \frac{1}{2}\right) \\ (1-x)^{\beta}, & x \in\left[\frac{1}{2}, 1\right)\end{cases}
$$

guarantees the decay needed to truncate the infinite quadrature rule. A less general but more convenient assumption than (3.12) is

$$
|E I(x) u(x)| \leq K x^{\alpha+\frac{3}{2}}(1-x)^{\beta+\frac{3}{3}} .
$$

With $\alpha$ and $\beta$ specified and $M_{x}$ chosen, the parameter selections

$$
h_{x}=\sqrt{\frac{\pi d}{\alpha M_{x}}}
$$


and

$$
N_{x}=\llbracket \frac{\alpha}{\beta} M_{x}+1 \rrbracket
$$

balance the asymptotic quadrature errors to at least $\mathcal{O}\left(e^{\left(-\pi d a M_{0}\right)^{\frac{1}{2}}}\right)$. This rate results from the presence of a sinc function in the integral. In the above, [.] denotes the greatest integer function. Note that if $\frac{\alpha}{\beta} M_{x}$ is an integer, (3.15) can be replaced by the selection $N_{x}=\frac{\alpha}{\bar{\beta}} M_{x}$.

The discrete system for (3.6), using the traditional approach, can then be formulated as follows. Let $I^{(\ell)}, \ell=0,1,2,3,4$ denote the $m_{x} \times m_{x}$ matrices whose pi-th entry is $\delta_{p i}^{(\ell)}$ from $(3.1)-(3.5)$ and let $\mathcal{D}(\eta)$ be the diagonal matrix with entries $\eta\left(x_{-M_{*}}\right), \cdots, \eta\left(x_{N_{*}}\right)$. The vector of unknowns $\vec{u}=\left[u_{-M_{0}}, \cdots, u_{N_{*}}\right]^{T}$ is then related to the known vector $\vec{f}=$ $\left[f\left(x_{-M_{\bullet}}\right), \cdots, f\left(x_{N_{\bullet}}\right)\right]^{T}$ by

$$
A_{x} \vec{u}=\mathcal{D}\left(\left(\phi^{\prime}\right)^{-\frac{k}{3}}\right) \vec{f}
$$

where

$$
A_{x}=\left[\frac{1}{h_{x}^{4}} I^{(4)} \mathcal{D}\left(a_{4}\right)+\frac{1}{h_{x}^{3}} I^{(3)} \mathcal{D}\left(a_{3}\right)+\frac{1}{h_{x}^{2}} I^{(2)} \mathcal{D}\left(a_{2}\right)+\frac{1}{h_{x}} I^{(1)} \mathcal{D}\left(a_{1}\right)+I^{(0)} \mathcal{D}\left(a_{0}\right)\right]
$$

The functions $a_{\ell}(x), \ell=0,1,2,3,4$ are given by

$$
\begin{gathered}
a_{0}=\frac{(E I w)^{\prime \prime \prime}}{\phi^{\prime}}-2 \frac{\left(E I^{\prime} w\right)^{\prime \prime \prime}}{\phi^{\prime}}+\frac{\left(E I^{\prime \prime} w\right)^{\prime \prime}}{\phi^{\prime}} \\
a_{1}=4(E I w)^{\prime \prime \prime}+6(E I w)^{\prime \prime} \frac{\phi^{\prime \prime}}{\phi^{\prime}}+4(E I w)^{\prime} \frac{\phi^{\prime \prime \prime}}{\phi^{\prime}}+E I w \frac{\phi^{\prime \prime \prime}}{\phi^{\prime}} \\
-6\left(E I^{\prime} w\right)^{\prime \prime}-6\left(E I^{\prime} w\right)^{\prime} \frac{\phi^{\prime \prime}}{\phi^{\prime}}-2 E I^{\prime} w \frac{\phi^{\prime \prime \prime}}{\phi^{\prime}}+E I^{\prime \prime}(w) \frac{\phi^{\prime \prime}}{\phi^{\prime}}+2\left(E I^{\prime \prime} w\right)^{\prime} \\
a_{2}=6(E I w)^{\prime \prime} 0^{\prime}+12(E I w)^{\prime} \phi^{\prime \prime}+4 E I w \phi^{\prime \prime \prime}+3(E I w)^{\prime} \frac{\left(\phi^{\prime \prime}\right)^{2}}{\phi^{\prime}} \\
-6\left(E I^{\prime} w\right)^{\prime} \phi^{\prime}-6 E I w \phi^{\prime \prime}+E I^{\prime \prime} w \phi^{\prime}, \\
a_{3}=4(E I w)^{\prime}\left(\phi^{\prime}\right)^{2}+6 E I w \phi^{\prime} \phi^{\prime \prime}-2 E I^{\prime} w\left(\phi^{\prime}\right)^{2}
\end{gathered}
$$

and

$$
a_{4}=E I w\left(\phi^{\prime}\right)^{3}
$$

Further details concerning the derivation of the system (3.16) and a thorough spectral analysis of the component matrices can be found in [13]. 
As mentioned previously, the treatment of the first integral in (3.10) yields various perturbations of the method which are advantageous in certain applications. One such application is the parameter recovery problem where an integral part of most numerical schemes for solving that problem is an accurate forward solver. With this in mind, the alternative approach mentioned above is implemented wherein the term $E I(x)$ in (3.10) is expanded as a linear combination of weighted sinc functions with four Hermite-like algebraic terms. These terms are added to accommodate the potentially nonzero function and derivative values of $E I$ at $x=0$ and $x=1$. Specifically, this parameter basis is taken to be $\left\{\psi_{k}\right\}_{k=-M_{*}}^{N_{*}}$ with

$$
\psi_{k}(x)= \begin{cases}b_{-M_{\bullet}}(x), & k=-M_{x} \\ b_{-M_{\bullet}+1}(x), & k=-M_{x}+1 \\ v_{E}(x) S_{k}(x), & -M_{x}+2 \leq k \leq N_{x}-2 \\ b_{N_{\bullet}-1}(x), & k=N_{x}-1 \\ b_{N_{-}}(x), & k=N_{x} .\end{cases}
$$

Here $S_{k}(x)=S\left(k, h_{x}\right) \circ \phi(x)$ and the basis weight $v_{E}$ is taken to be

$$
v_{E}(x)=w(x)=[x(1-x)]^{\frac{1}{2}}
$$

The algebraic boundary basis functions are given by

$$
\begin{gathered}
b_{-M_{-}+1}(x)=(1-x)^{2}[2 x+1] \\
b_{N_{\bullet}-1}(x)=x^{2}[2(1-x)+1] \\
b_{-M_{\bullet}}(x)=x(1-x)^{2}
\end{gathered}
$$

and

$$
b_{N_{0}}(x)=-x^{2}(1-x)
$$

The finite dimensional approximation of EI then takes the form

$$
E I_{m_{\bullet}}(x)=\sum_{k=-M_{\bullet}}^{N_{\bullet}} c_{k} \psi_{k}(x)
$$


The number of basis functions used in the expansion is chosen so as to guarantee a square coefficient matrix. This is done to simplify the implementation of the method when applied to the PDE (1.1) of interest.

A quick note should be made concerning the choice of basis and the manner of expanding $E I_{m_{\text {. }}}$. The two derivative-interpolating boundary basis functions are added so that this expansion of $E I_{m_{\varepsilon}}$ is the same as that used with cantilever or free boundary conditions. The choice of (3.24) for basis weight is certainly sufficient and proves to be beneficial when incorporating this forward scheme into a numerical method for solving the parameter recovery problem.

The expansion (3.25) is substituted into (3.10) and the resulting integrals are evaluated via Theorem 2.2 or Theorem 2.1 when possible. The decay condition (2.4) equates to the condition

$$
|\mathcal{E} \mathcal{I}(x) u(x)| \leq \hat{K} \begin{cases}x^{\alpha+\frac{3}{3}}, & x \epsilon\left(0, \frac{1}{2}\right) \\ (1-x)^{\beta+\frac{3}{2}}, & x \epsilon\left(\frac{1}{2}, 1\right)\end{cases}
$$

where the "homogeneous" part of $\mathrm{EI}$ is

$$
\mathcal{E} I(x)=E I(x)-E I(0) b_{-M_{\bullet}+1}(x)-E I(1) b_{N_{z}-1}(x)-E I^{\prime}(0) b_{-M_{\bullet}}(x)-E I^{\prime}(1) b_{N_{\bullet}}(x) \text {. }
$$

The arguments leading to this condition are analogous to those presented in the second-order case as described in [14]. Again, this may be replaced by the more stringent requirement

$$
|\mathcal{E} \mathcal{I}(x) u(x)| \leq K x^{\alpha+\frac{1}{2}}(1-x)^{\beta+\frac{1}{2}} .
$$

As before, the asymptotic errors are balanced by choosing $h_{x}$ and $N_{x}$ as specified in (3.14) and (3.15).

With $\vec{u}$ and $\vec{f}$ defined as before and EI expanded, the system for (3.6) using this alternative approach can be written as

$$
A_{x} \vec{u}=\mathcal{D}\left(\left(\phi^{\prime}\right)^{-\frac{b}{2}}\right) \vec{f}
$$

where

$$
A_{x}=\left[\Phi^{(2)} \mathcal{D}\left(\vec{p}_{\psi^{(2)}}\right)+2 \Phi^{(3)} \mathcal{D}\left(\vec{p}_{\psi(1)}\right)+\Phi^{(4)} \mathcal{D}\left(\vec{p}_{\psi(0)}\right)\right]
$$


The notation $\mathcal{D}\left(\vec{p}_{\psi}(\ell)\right), \ell=0,1,2$ denotes the diagonal matrices containing the components of the vectors

$$
\vec{p}_{\Psi(\ell)}=\Psi^{(\ell)} \vec{c}, \quad \ell=0,1,2
$$

where $\vec{c}=\left[c_{-M_{\bullet}}, \cdots, c_{N_{\bullet}}\right]^{T}$. The matrices $\Phi^{(j)}, j=2,3,4$ and $\Psi^{(\ell)}, \ell=0,1,2$ are defined componentwise by

$$
\left[\Phi^{(j)}\right]_{p i}=\frac{1}{\phi^{\prime}\left(x_{i}\right)}\left(S_{p} w\right)^{(j)}\left(x_{i}\right)
$$

and

$$
\left[\Psi^{(\ell)}\right]_{i k}=\psi_{k}^{(l)}\left(x_{i}\right)
$$

The notation on the right-hand sides of (3.31) and (3.32) indicates the $\mathrm{j}$-th and $\ell$-th derivatives, respectively.

To illustrate the dependence of $\Phi^{(j)}, j=2,3,4$ and $\Psi^{(\ell)}, \ell=0,1,2$ on previously defined matrices, the respective expansions are listed below. The diagonal matrices $\mathcal{D}$ and the matrices $I^{(\ell)}, \ell=0,1,2,3,4$ have sizes consistent with the following range of the indices $i, p$ and $k\left(-M_{x} \leq i \leq N_{x},-M_{x} \leq p \leq N_{x},-M_{x}+2 \leq k \leq N_{x}-2\right)$. From (3.31) it follows that

$$
\begin{aligned}
\Phi^{(2)}= & \frac{1}{h_{x}^{2}} I^{(2)} \mathcal{D}\left(w \phi^{\prime}\right)+\frac{1}{h_{x}} I^{(1)} \mathcal{D}\left(2 w^{\prime}\right)+I^{(0)} \mathcal{D}\left(w^{\prime \prime}\right), \\
\Phi^{(3)}= & \frac{1}{h_{x}^{3}} I^{(3)} \mathcal{D}\left(w\left(\phi^{\prime}\right)^{2}\right)+\frac{1}{h_{x}^{2}} I^{(2)} \mathcal{D}\left(3 w^{\prime} \phi^{\prime}+3 w \phi^{\prime \prime}\right) \\
& +\frac{1}{h_{x}} I^{(1)} \mathcal{D}\left(3 w^{\prime \prime}+3 w^{\prime} \frac{\phi^{\prime \prime}}{\phi^{\prime}}+w \frac{\phi^{\prime \prime \prime}}{\phi^{\prime}}\right)+I^{(0)} \mathcal{D}\left(\frac{w^{\prime \prime \prime}}{\phi^{\prime}}\right)
\end{aligned}
$$

and

$$
\begin{aligned}
\Phi^{(4)}= & \frac{1}{h_{x}^{4}} I^{(4)} \mathcal{D}\left(w\left(\phi^{\prime}\right)^{3}\right)+\frac{1}{h_{x}^{3}} I^{(3)} \mathcal{D}\left(4 w^{\prime}\left(\phi^{\prime}\right)^{2}+6 w \phi^{\prime} \phi^{\prime \prime}\right) \\
& +\frac{1}{h_{x}^{2}} I^{(2)} \mathcal{D}\left(6 w^{\prime \prime} \phi^{\prime}+12 w^{\prime} \phi^{\prime \prime}+4 w \phi^{\prime \prime \prime}+3 w \frac{\left(\phi^{\prime \prime}\right)^{2}}{\phi^{\prime}}\right) \\
& +\frac{1}{h_{x}} I^{(1)} \mathcal{D}\left(4 w^{\prime \prime \prime}+6 w^{\prime \prime} \frac{\phi^{\prime \prime}}{\phi^{\prime}}+4 w^{\prime} \frac{\phi^{\prime \prime \prime}}{\phi^{\prime}}+w \frac{\phi^{\prime \prime \prime}}{\phi^{\prime}}\right) \\
& +I^{(0)} \mathcal{D}\left(\frac{w^{\prime \prime \prime}}{\phi^{\prime}}\right) .
\end{aligned}
$$

For $\ell=0,1,2$ the $m_{x} \times m_{x}$ matrices in (3.32) are given by

$$
\Psi^{(\ell)}=\left[\vec{b}_{-M_{\bullet}}^{(\ell)} \vdots \vec{b}_{-M_{\bullet}+1}^{(\ell)} \vdots B^{(\ell)} \vdots \vec{b}_{N_{\bullet}-1}^{(\ell)} \vdots \vec{b}_{N_{\bullet}}^{(\ell)}\right]
$$


where $\vec{b}_{k}^{(\ell)}=\left[b_{k}^{(\ell)}\left(x_{-M_{*}}\right), \cdots, b_{k}^{(\ell)}\left(x_{N_{*}}\right)\right]^{T}$ for $k=-M_{x},-M_{x}+1, N_{x}-1$, and $N_{x}$. Again, the superscript $\ell$ indicates the $\ell$-th derivative. The $m_{x} \times\left(m_{x}-4\right)$ matrices $B^{(\ell)}$ are

$$
\begin{gathered}
B^{(0)}=\mathcal{D}\left(v_{E}\right) I^{(0)} \\
B^{(1)}=-\frac{1}{h_{x}} \mathcal{D}\left(v_{E} \phi^{\prime}\right) I^{(1)}+\mathcal{D}\left(v_{E}\right) I^{(0)}
\end{gathered}
$$

and

$$
B^{(2)}=\frac{1}{h_{x}^{2}} \mathcal{D}\left(v_{E}\left(\phi^{\prime}\right)^{2}\right) I^{(2)}-\frac{1}{h_{x}} \mathcal{D}\left(v_{E} \phi^{\prime \prime}+2 v_{E}^{\prime} \phi^{\prime}\right) I^{(1)}+\mathcal{D}\left(v_{E}^{\prime \prime}\right) I^{(0)} .
$$

The negative signs that appear in the definitions of $B^{(1)}$ and $B^{(2)}$ result from the transposing of $I^{(1)}$. Again, it is noted that in (3.37) - (3.39), the $m_{x} \times\left(m_{x}-4\right)$ matrices $I^{(\ell)}, \ell=0,1,2$ have components $\delta_{i k}^{(\ell)}$ as defined in (3.1) - (3.3).

Thus, the fourth-order spatial problem (3.6) can be solved in a variety of ways using the Sinc-Galerkin method. For standard forward problems, the system (3.16) is often the most convenient to formulate and solve. If the forward solver is part of a numerical routine for solving the parameter recovery problem, then (3.28) is more useful since $E I$ is replaced by its finite dimensional approximation. Both approaches yield solutions $u_{m}$. which are exponentially convergent approximations to the solution $u$ of (3.6).

\subsection{The Spatial Problem: Cantilever Boundary Conditions}

A second set of fourth-order boundary conditions arise when modeling beams that are fixed at one end and free at the other. To extend the Sinc-Galerkin method to problems with these cantilever boundary conditions, consider the ODE

$$
\begin{array}{ll}
L u(x)=\left(E I(x) u^{\prime \prime}(x)\right)^{\prime \prime} & =f(x), 0<x<1 \\
u(0)=\bar{\alpha}, & \left(E I u^{\prime \prime}\right)(1)=\bar{\gamma} \\
u^{\prime}(0)=\bar{\beta}, & \left(E I u^{\prime \prime}\right)^{\prime}(1)=\bar{\delta} .
\end{array}
$$

A Sinc-Galerkin method to approximate the solution of (3.40) can be developed as follows. 
Define the set of basis functions $\left\{\zeta_{i}\right\}_{i=-M_{0}-4}^{N_{0}+4}$ by

$$
\zeta_{i}(x)= \begin{cases}B_{-M_{\bullet}-4}(x), & i=-M_{x}-4 \\ B_{-M_{\bullet}-3}(x), & i=-M_{x}-3 \\ B_{-M_{\bullet}-2}(x), & i=-M_{x}-2 \\ B_{-M_{\bullet}-1}(x), & i=-M_{x}-1 \\ v(x) S_{i}(x), & -M_{x} \leq i \leq N_{x} \\ B_{N_{\bullet}+1}(x), & i=N_{x}+1 \\ B_{N_{\bullet}+3}(x), & i=N_{x}+2 \\ B_{N_{+}+3}(x), & i=N_{x}+3 \\ B_{N_{\bullet}+4}(x), & i=N_{x}+4 .\end{cases}
$$

Here $S_{i}(x) \equiv S\left(i, h_{x}\right) \circ \phi(x)$ and the basis weight $v(x)$ is taken to be

$$
v(x)=[x(1-x)]^{3} .
$$

The boundary basis functions are

$$
\begin{gathered}
B_{-M_{\bullet}-1}(x)=(1-x)^{4}\left[20 x^{3}+10 x^{2}+4 x+1\right], \\
B_{N_{\bullet}+1}(x)=x^{4}\left[20(1-x)^{3}+10(1-x)^{2}+4(1-x)+1\right], \\
B_{-M_{\bullet}-2}(x)=x(1-x)^{4}\left[10 x^{2}+4 x+1\right], \\
B_{N_{\bullet}+2}(x)=-x^{4}(1-x)\left[10(1-x)^{2}+4(1-x)+1\right], \\
B_{-M_{\bullet}-3}(x)=x^{2}(1-x)^{4}\left[2 x+\frac{1}{2}\right], \\
B_{N_{\bullet}+3}(x)=x^{4}(1-x)^{2}\left[2(1-x)+\frac{1}{2}\right], \\
B_{-M_{\bullet}-4}(x)=\frac{1}{6} x^{3}(1-x)^{4}
\end{gathered}
$$

and

$$
B_{N_{*}+4}(x)=-\frac{1}{6} x^{4}(1-x)^{3}
$$


A brief note concerning the choice of basis is in order at this point. First, since $\frac{\left.d^{\ell}\right)}{d x^{(l)}}\left[S\left(i, h_{x}\right) \circ \phi(x)\right], \ell=1,2, \cdots$, are undefined at $x=0$ and $x=1$, some basis modifications must be made when solving problems with nonzero boundary conditions (see also the definition of $\psi_{k}$ in (3.23)). It is tempting to use fewer algebraic boundary basis functions and the basis weight

$$
v(x)=x(1-x)^{3},
$$

but in many problems this results in nonzero boundary terms when integrating by parts. By using the basis weight $v(x)=[x(1-x)]^{3}$ and a full complement of algebraic terms, this pitfall can be avoided. Furthermore, the basis $\left\{\zeta_{i}\right\}$ as defined in (3.41) can be used for problems with free boundary conditions, thus providing consistency to the method.

The approximate solution is then defined to be

$$
\begin{aligned}
u_{m_{\varepsilon}}(x)= & \bar{\alpha} \zeta_{-M_{\bullet}-1}(x)+\bar{\beta} \zeta_{-M_{\bullet}-2}(x)+\tilde{\gamma} \zeta_{N_{\bullet}+3}(x)+\tilde{\delta} \zeta_{N_{\bullet}+4}(x) \\
& +u_{-M_{\bullet}-3} \zeta_{-M_{\bullet}-3}(x)+u_{-M_{\bullet}-4} \zeta_{-M_{\bullet}-4}(x)+u_{N_{\bullet}+1} \zeta_{N_{\bullet}+1}(x) \\
& u_{N_{\bullet}+2} \zeta_{N_{\bullet}+2}(x) \\
& +\sum_{i=-M_{x}}^{N_{\bullet}} u_{i} \zeta_{i}(x)
\end{aligned}
$$

where $\bar{\alpha}, \bar{\beta}, \tilde{\gamma}$, and $\bar{\delta}$ are known and the coefficients $\left\{u_{i}\right\}$ are unknown. The quantities

$$
\tilde{\gamma} \equiv \frac{1}{E I(1)} \bar{\gamma}
$$

and

$$
\tilde{\delta} \equiv \frac{1}{E I(1)} \bar{\delta}-\frac{E I^{\prime}(1)}{[E I(1)]^{2}} \bar{\gamma}
$$

are well-defined since $E I(x)$ is assumed positive on $[0,1]$. Note that with the definition (3.41) for the basis $\left\{\zeta_{i}\right\}, u_{m_{-}}(x)$ satisfies the boundary conditions; that is,

$$
\begin{aligned}
& u_{m_{\bullet}}(0)=\bar{\alpha}, \quad\left(E I u_{m_{\bullet}}^{\prime \prime}\right)(1)=\bar{\gamma} \\
& u_{m_{\bullet}}^{\prime}(0)=\bar{\beta}, \quad\left(E I u_{m_{\Xi}}^{\prime \prime}\right)^{\prime}(1)=\bar{\delta}
\end{aligned}
$$

The $m_{x}+4$ unknown coefficients in (3.43) are determined by orthogonalizing the residual with respect to the set of sinc functions $\left\{S_{p}\right\}_{p=-M_{*}-2}^{N_{s}+2}$. This Petrov-Galerkin approach is in 
contrast to those Galerkin methods in which the residual is orthognalized with respect to the basis and is done to take advantage of the exponential accuracy of point evaluation in the quadratures. This yields the discrete system

$$
\left(L u_{m_{\bullet}}-f, S_{p}\right)=0
$$

for $p=-M_{x}-2, \cdots, N_{x}+2$. The inner product $(\cdot, \cdot)$ is that defined in (3.8) with the weight in this case taken to be

$$
w(x)=1 .
$$

The difference between the weight function in (3.45) and that given in (3.9) is due to the presence of the basis weight $v(x)$ in the definition of the basis $\left\{\zeta_{i}\right\}$. If the definition

$$
u_{h}(x)=\sum_{i=-M_{\bullet}}^{N_{a}} u_{i} \zeta_{i}(x)
$$

is made, then (3.41) and (3.44) can be combined to yield

$$
\begin{aligned}
& \left(L B_{-M_{\bullet}-3}, S_{p}\right) u_{-M_{\bullet}-3}+\left(L B_{-M_{\bullet}-4}, S_{p}\right) u_{-M_{*}-4}+\left(L B_{N_{*}+1}, S_{p}\right) u_{N_{*}+1} \\
& +\left(L B_{N_{*}+2}, S_{p}\right) u_{N_{*}+2}+\left(L u_{h}, S_{p}\right) \\
& =\left(f, S_{p}\right)
\end{aligned}
$$

for $p=-M_{x}-2, \cdots, N_{x}+2$ and

$$
\bar{f}(x) \equiv f(x)-\bar{\alpha} L B_{-M_{\bullet}-1}(x)-\bar{\beta} L B_{-M_{\bullet}-2}(x)-\tilde{\gamma} L B_{N_{e}+3}(x)-\tilde{\delta} L B_{N_{\bullet}+4}(x)
$$

Integration by parts is applied to $\left(L u_{h}, S_{p}\right)$ thus yielding the integral

$$
\int_{0}^{1} u_{h}(x)\left[E I(x)\left(S_{p}(x) w(x)\right)^{\prime \prime}\right]^{\prime \prime} d x
$$

(compare to (3.10)). The weight choice (3.45) is sufficient for guaranteeing that the boundary term (3.11) vanishes with $u$ replaced by $u_{h}$. As in Section 3.1 there are two approaches here. In the traditional approach $E I(x)$ is differentiated directly and in the alternative approach $E I$ is simply replaced by the finite dimensional approximate $E I_{m_{*}}$. The remaining inner products in (3.46) are evaluated directly via Theorem 2.1. 
Proceeding with the first approach, as indicated by (3.12), the choice of weight $w$ directly affects the decay conditions dictated by (2.9) of Theorem 2.2. For the weight $w(x)=1$ the condition

$$
|E I(x) \mathcal{U}(x)| \leq x^{\alpha+3}(1-x)^{\beta+3}
$$

guarantees sufficient decay so that the asymptotic errors resulting from the quadrature can be balanced by choosing $h_{x}$ and $N_{x}$ as specified in (3.14) and (3.15). The term $\mathcal{U}(x)$ denotes that part of the true solution which is approximated by $u_{h}$ and is given by the formal change of variables

$$
\begin{aligned}
\mathcal{U}(x)= & u(x)-\bar{\alpha} B_{-M_{\bullet}-1}(x)-\bar{\beta} B_{-M_{\bullet}-2}(x)-\tilde{\gamma} B_{N_{\bullet}+3}(x)-\tilde{\delta} B_{N_{\bullet}+4}(x) \\
& -u^{\prime \prime}(0) B_{-M_{\bullet}-3}(x)-u^{\prime \prime \prime}(0) B_{-M_{\bullet}-4}(x) \\
& -u(1) B_{N_{\bullet}+1}(x)-u^{\prime}(1) B_{N_{+}+2}(x) .
\end{aligned}
$$

The discrete system for (3.40) can then be formulated as follows. Let $\vec{a}_{-M_{-}-3}, \vec{a}_{-M_{-}-4}$, $\vec{a}_{N_{*}+1}, \vec{a}_{N_{\bullet}+2}$ and $\vec{f}$ denote the $\left(m_{x}+4\right) \times 1$ vectors containing the product of $\frac{1}{h_{0}}$ and the approximations to the inner products $\left(L B_{-M_{--3}}, S_{p}\right),\left(L B_{-M_{0-4}}, S_{p}\right),\left(L B_{-N_{m}+1}, S_{p}\right)$, $\left(L B_{N_{*}+2}, S_{p}\right)$, and $\left(\mathcal{f}, S_{p}\right)$, respectively. Hence the p-th entries of the respective vectors are

$$
\begin{aligned}
& \left(\vec{a}_{i}\right)_{p}=\left(E I B_{i}^{\prime \prime}\right)^{\prime \prime}\left(x_{p}\right) \frac{w\left(x_{p}\right)}{\phi^{\prime}\left(x_{p}\right)} \\
& \left(i=-M_{x}-3,-M_{x}-4, N_{x}+1, N_{x}+2\right) \text { and } \\
& (\vec{f})_{p}=\bar{f}\left(x_{p}\right) \frac{w\left(x_{p}\right)}{\phi^{\prime}\left(x_{p}\right)} .
\end{aligned}
$$

Furthermore, let $A_{m}$ denote the $\left(m_{x}+4\right) \times m_{x}$ matrix which results from the expansion of the inner product $\left(L u_{h}, S_{p}\right)$. For $a_{\ell}(x), \ell=0,1,2,3,4$ as defined in (3.18) - (3.22), the matrix $A_{m}$ is given by

$$
\begin{aligned}
A_{m}= & \frac{1}{h_{x}^{4}} I^{(4)} \mathcal{D}\left(v a_{4}\right)+\frac{1}{h_{x}^{3}} I^{(3)} \mathcal{D}\left(v a_{3}\right)+\frac{1}{h_{x}^{2}} I^{(2)} \mathcal{D}\left(v a_{2}\right) \\
& +\frac{1}{h_{x}} I^{(1)} \mathcal{D}\left(v a_{1}\right)+I^{(0)} \mathcal{D}\left(v a_{0}\right)
\end{aligned}
$$

Here $I^{(\ell)}, \ell=0,1,2,3,4$ are $\left(m_{x}+4\right) \times m_{x}$ matrices whose $p i$-th entry is given by $\delta_{p i}^{(\ell)}$ from (3.1) - (3.5), and $v$ is defined in (3.42). 
The discrete system for the determination of the unknown coefficients $\left\{u_{i}\right\}$ is given by

$$
A_{x} \vec{u}=\vec{f}
$$

where the $\left(m_{x}+4\right) \times\left(m_{x}+4\right)$ matrix $A_{x}$ is defined to be

$$
A_{x}=\left[\vec{a}_{-M_{0}-4} \vdots \vec{a}_{-M_{\bullet}-3} \vdots A_{m} \vdots \vec{a}_{N_{*}+1} \vdots \vec{a}_{N_{e}+2}\right] .
$$

Here $\vec{u}$ is defined to be the $\left(m_{x}+4\right) \times 1$ vector

$$
\vec{u}=\left[u_{-M_{\bullet}-4}, u_{-M_{\bullet}-3}, u_{-M_{-}}, \cdots, u_{N_{-}}, u_{N_{\bullet}+1}, u_{N_{-}+2}\right]^{T}
$$

containing the unknowns.

It is noted that the matrices $A_{x}$ as defined in (3.17) and (3.50) differ only in the presence of $v$ in the diagonal multipliers and the addition of border vectors. Hence the method is easily adapted when the boundary conditions are changed. Moreover, the exponential convergence rate is maintained, thus preserving the accuracy of the method.

With parameter recover in mind, it is again worthwhile to use the alternative approach to develop the discrete system which arises when $E I(x)$ is replaced by the finite dimensional term $E I_{m_{\Delta}}$ as defined in (3.25). To simplify notation in the discussion which follows, let

$$
E I_{h}(x)=\sum_{k=-M_{\bullet}}^{N_{\bullet}} c_{k} v_{E}(x) S_{k}(x)
$$

and

$$
E I_{c}(x)=c_{-M_{\bullet}-1} b_{-M_{-}-1}(x)+c_{-M_{\bullet-2}} b_{-M_{e-2}}(x)+c_{N_{\bullet}+1} b_{N_{\bullet}+1}(x)+c_{N_{+}+2} b_{N_{-}+2}(x)
$$

so that $E I_{m_{m}}=E I_{h}+E I_{c}$. Note that $E I_{h}$ and $E I_{c}$ simply denote the sinc and algebraic components of the approximate parameter.

Consider now the inner products found in the system (3.46). The expansion of $\left(L u_{h}, S_{p}\right)$ proceeds exactly as before with $E I(x)$ simply being replaced by $E I_{m_{a}}(x)$ in the integral (3.47). In the boundary inner products, $\left(L B_{i}, S_{p}\right), i=-M_{x}-4, \cdots,-M_{x}-1$ and $i=N_{x}+1$, $\cdots, N_{x}+4$, expansion and integration by parts yields

$$
\begin{aligned}
\left(L B_{i}, S_{p}\right)= & \left\{\int_{0}^{1} E I_{h}(x) B_{i}^{\prime \prime}(x)\left(S_{p} w\right)^{\prime \prime}(x) d x+B_{T}\right\} \\
& +\int_{0}^{1}\left(E I_{c} B_{i}^{\prime \prime}\right)^{\prime \prime}(x) S_{p}(x) w(x) d x .
\end{aligned}
$$


The weight $w(x)=1$ (see (3.45)) is sufficient for guaranteeing that

$$
B_{T}=\left.\left\{\left(E I_{h} B_{i}^{\prime \prime}\right)^{\prime}\left(S_{p} w\right)-E I_{h} B_{i}^{\prime \prime}\left(S_{p} w\right)^{\prime}\right\}(x)\right|_{0} ^{1}=0 .
$$

The resulting integrals are evaluated via Theorem 2.2 or, when possible, Theorem 2.1.

For the weight $w(x)=1$, the decay condition is

$$
|\mathcal{E} \mathcal{I}(x) \mathcal{U}(x)| \leq x^{\alpha+3}(1-x)^{\beta+3}
$$

where $\mathcal{U}$ and $\mathcal{E} I$ are defined in (3.49) and (3.27) (compare to (3.48)). Again, the asymptotic errors are balanced by choosing $h_{x}$ and $N_{x}$ as specified in (3.14) and (3.15).

The matrix system corresponding to (3.40) may be formulated as follows. Let $\Phi^{(j)}, \Psi^{(\ell)}$, and $\vec{p}_{\Psi(\ell)}$ be defined as they were in (3.31), (3.32), and (3.30), respectively (with $\ell=0,1,2$ and $j=2,3,4)$. Note that in the definitions now, the index ranges are $-M_{x} \leq i \leq N_{x}$, $-M_{x}-2 \leq p \leq N_{x}+2$, and $-M_{x}-2 \leq k \leq N_{x}+2$, and the $\left(m_{x}+4\right) \times 1$ coefficient vector is now $\vec{c}=\left[c_{-M_{*}-2}, \cdots, c_{N_{0}+2}\right]^{T}$. Hence $\Phi^{(j)}, \Psi^{(\ell)}$ and $\vec{p}_{\Psi}(\ell)$ have the sizes $\left(m_{x}+4\right) \times m_{x}$, $m_{x} \times\left(m_{x}+4\right)$ and $m_{x} \times 1$, respectively.

Furthermore, let $\hat{\Phi}^{\prime \prime}$ denote the $\left(m_{x}+4\right) \times m_{x}$ matrix which is defined componentwise by

$$
\left[\hat{\Phi}^{\prime \prime}\right]_{p k}=\frac{1}{\phi^{\prime}\left(x_{k}\right)}\left(S_{p} w\right)^{\prime \prime}\left(x_{k}\right)
$$

and let $\tilde{c}=\left[c_{-M_{\varepsilon}}, \cdots, c_{N_{\varepsilon}}\right]^{T}$. Finally, for $i=-M_{x}-4, \cdots,-M_{x}-1$ and $i=N_{x}+1, \cdots, N_{x}+4$, let $\vec{a}_{i}$ denote the $\left(m_{x}+4\right) \times 1$ vectors

$$
\vec{a}_{i}=\left[\hat{\Phi}^{\prime \prime} \mathcal{D}\left(v_{E} B_{i}^{\prime \prime}\right) \tilde{c}+\mathcal{D}\left(\frac{w}{\phi^{\prime}}\left(E I_{c} B_{i}^{\prime \prime}\right)^{\prime \prime}\right) \overrightarrow{1}\right] .
$$

Here $\overrightarrow{1}$ is simply the $m_{x} \times 1$ vector of ones.

With the unknown vector $\vec{u}$ given by (3.51), the discrete system can be written as

$$
A_{x} \vec{u}=\vec{f}
$$

where

$$
A_{x}=\left[\vec{a}_{-M_{\bullet-4}} \vdots \vec{a}_{-N_{\bullet}-3} \vdots A_{m} \vdots \vec{a}_{N_{\bullet}+1} \vdots \vec{a}_{N_{\bullet+2}}\right]
$$

and

$$
\vec{f}=\mathcal{D}\left(\frac{w}{\phi^{\prime}} f\right) \overrightarrow{1}-\bar{\alpha} \vec{a}_{-M_{*}-1}-\vec{\beta} \vec{a}_{-M_{\bullet-2}}-\tilde{\gamma} \vec{a}_{N+3}-\tilde{\delta} \vec{a}_{N+4}
$$


The $\left(m_{x}+4\right) \times m_{x}$ submatrix $A_{m}$ is given by

$$
A_{m}=\left[\Phi^{(2)} \mathcal{D}\left(v \vec{p}_{\Psi(2)}\right)+2 \Phi^{(3)} \mathcal{D}\left(v \vec{p}_{\Psi(1)}\right)+\Phi^{(4)} \mathcal{D}\left(v \vec{p}_{\Psi(0)}\right)\right]
$$

It should be noted that the coefficient matrix $A_{x}$ in (3.52) differs from that arising in the fixed boundary problem, (3.29), only in the presence of $v$ in the diagonal multipliers and the addition of border vectors. This makes the method easily adaptable when changing the boundary conditions. Furthermore, the matrices $\Phi^{(j)}, \hat{\Phi}^{\prime \prime}$, and $\Psi^{(\ell)}$ can be expanded in terms of fundamental matrices in a manner similar to that in (3.33) - (3.36), thus simplifying the implementation of the method. Finally, the exponential convergence rate of the method is maintained, thus preserving the method's accuracy.

With the techniques from this section, the implementation of the Sinc-Galerkin method for problems with free and simple boundary conditions can be accomplished in a manner that is completely analogous to that used for cantilever boundary conditions. Further details and examples of the Sinc-Galerkin method for problems with free and simple boundary conditions can be found in [8].

\subsection{The Temporal Problem}

The last ODE to be considered is the initial value problem

$$
\begin{aligned}
& P u(t) \equiv \ddot{u}(t)=f(t), \quad 0<t<\infty \\
& u(0)=\dot{u}(0)=0
\end{aligned}
$$

A Sinc-Galerkin method to approximate the solution of (3.53) can be developed in a manner similar to that of the preceding boundary value problems. Define the set of basis functions $\left\{S_{j}^{*}\right\}_{j=-M_{i}}^{N_{t}}$ by

$$
S_{j}^{*}(t)=S\left(j, h_{t}\right) \circ \Upsilon(t)
$$

where $\Upsilon: D_{W} \rightarrow D_{S}$ is given in (2.2). The approximate solution $u_{m_{t}}(t)$ is then defined by

$$
u_{m_{t}}(t)=\sum_{j=-M_{t}}^{N_{t}} u_{j} S_{j}^{*}(t), \quad m_{t}=M_{t}+N_{t}+1
$$

The $m_{t}$ unknown coefficients $\left\{u_{j}\right\}$ in (3.54) are determined by orthogonalizing the residual 
with respect to the set of sinc functions $\left\{S_{q}^{*}\right\}_{q=-M_{t}}^{N_{t}}$ which leads to the analysis of

$$
\begin{aligned}
\left(f, S_{q}^{*}\right) & =\left(P u, S_{q}^{*}\right) \\
& =\left(\ddot{u}, S_{q}^{*}\right)
\end{aligned}
$$

for $q=-M_{t}, \cdots, N_{t}$. The weighted inner product for (3.55) is defined to be

$$
(F, G)=\int_{0}^{\infty} F(t) G(t) w^{*}(t) d t
$$

and the weight is taken to be

$$
w^{*}(t)=\frac{1}{\sqrt{\dot{\Upsilon}(t)}}
$$

for reasons that are discussed in [4]. As before, integration by parts is used to transfer the differentiation of $u$ onto $S^{*} w^{*}$. To guarantee that boundary terms vanish, it is assumed that

$$
\lim _{i \rightarrow 0} \frac{\dot{u}(t) \sqrt{t}}{\ln (t)}=0
$$

The resulting integrals are then evaluated via the quadrature rules of Section 2. With respect to $(2.4)$, the condition

$$
|u(t) \sqrt{\dot{\Upsilon}(t)}| \leq \hat{K} \begin{cases}t^{\gamma}, & t \in(0,1) \\ t^{-\delta}, & t \in[1, \infty)\end{cases}
$$

guarantees the boundedness necessary to truncated the infinite quadrature rule. With $\gamma$ and $\delta$ specified and $M_{t}$ chosen, the parameter selections

$$
h_{t}=\sqrt{\frac{\pi d}{\gamma M_{t}}}
$$

and

$$
N_{t}=\llbracket \frac{\gamma}{\delta} M_{t}+1 \rrbracket
$$

balance the asymptotic errors in (2.5) to at least $O\left(e^{-\left(\pi d \gamma M_{t}\right)}\right)$.

In many time-dependent PDE's, it is reasonable to assume that the solution decays exponentially at infinity; that is, the solution satisfies

$$
|u(t) \sqrt{\dot{\Upsilon}(t)}| \leq \hat{K} \begin{cases}t^{\gamma}, & t \in(0,1) \\ e^{-\delta t}, & t \in[1, \infty)\end{cases}
$$


or, more stringently,

$$
|u(t)| \leq K t^{\gamma+\frac{1}{2}} e^{-\delta t} .
$$

With this supposition, Lund [12] shows that the condition (3.56) can be replaced by

$$
\left.N_{t}=\llbracket \frac{1}{h_{t}} \ln \left(\frac{\gamma}{\delta} M_{t} h_{t}\right)+1\right] .
$$

The selection $N_{t}$ in (3.58) significantly reduces the size of the discrete system with no loss of accuracy.

The discrete system for (3.53) can then be formulated as follows. Let $I^{(\ell)}, \ell=0,2$ denote the $m_{t} \times m_{t}$ matrices whose $q j$-th entry is $\delta_{q j}^{(\ell)}$ from (3.1) and (3.3), and let $\mathcal{D}(\eta)$ again be the diagonal matrix with entries $\eta\left(t_{-M_{t}}\right), \cdots, \eta\left(t_{N_{t}}\right)$. With the usual definitions for $\vec{u}$ and $\vec{f}$ and the identity

$$
(\dot{\Upsilon}(t))^{-\frac{1}{2}}\left\{(\dot{\Upsilon}(t))^{-\frac{1}{2}}\right\}^{\prime \prime}=-\frac{1}{4},
$$

the system for the determination of the unknown coefficients $\left\{u_{j}\right\}$ is given by

$$
A_{\imath} \vec{u}=\mathcal{D}\left((\dot{\Upsilon})^{-\frac{3}{2}}\right) \vec{f}
$$

where

$$
A_{t}=\left[\frac{1}{h_{t}^{2}} I^{(2)}-\frac{1}{4} I\right] \mathcal{D}\left((\dot{\Upsilon})^{\frac{1}{5}}\right) .
$$

Further details concerning the derivation of the system (3.59) can be found in [4].

Note that nonzero initial conditions can be handled in a manner analogous to that used for nonzero boundary conditions in the previous discussion. Rational initial basis functions are used to incorporate the initial behavior and this known contribution is then taken to the right-hand side of the resulting discrete system (see [8]). All other analysis is identical to that for the problem (3.53).

\section{Time-Dependent Problems}

This section details the Sinc-Galerkin method applied to fourth-order time-dependent PDE's with fixed and cantilever boundary conditions. Since the choice of basis, test functions, and inner product are all straightforward extensions of those used to solve the ODE's in Section 3, 
the error analysis and system formulation follow directly from previously discussed results. Once a discrete system has been formulated, various options exist for solving the associated matrix equation. Two such algorithms are outlined and their relative merits for various problems are discussed.

\subsection{The Time-Dependent Problem: Fixed Boundary Conditions}

Consider the time-dependent problem

$$
\begin{gathered}
\mathcal{L} u(x, t) \equiv \frac{\partial^{2} u}{\partial t^{2}}(x, t)+\frac{\partial^{2}}{\partial x^{2}}\left(E I(x) \frac{\partial^{2} u}{\partial x^{2}}(x, t)\right)=f(x, t), \quad 0<x<1 \quad t>0 \\
u(0, t)=u(1, t)=0, \quad t>0 \\
\frac{\partial u}{\partial x}(0, t)=\frac{\partial u}{\partial x}(1, t)=0, \quad t>0 \\
u(x, 0)=\frac{\partial u}{\partial t}(x, 0)=0, \quad 0 \leq x \leq 1 .
\end{gathered}
$$

Given the basis $\left\{S_{i j}\right\}$ where

$$
S_{i j}(x, t) \equiv S_{i}(x) S_{j}^{\dagger}(t)=S\left(i, h_{x}\right) \circ \phi(x) S\left(j, h_{t}\right) \circ \Upsilon(t)
$$

the approximate solution is defined by way of the tensor product expansion

$$
u_{m_{\bullet} m_{\mathrm{t}}}(x, t)=\sum_{i=-M_{\mathrm{e}}}^{N_{\mathrm{e}}} \sum_{j=-M_{t}}^{N_{t}} u_{i j} S_{i j}(x, t), \quad m_{x}=M_{x}+N_{x}+1, \quad m_{t}=M_{t}+N_{t}+1 .
$$

The $m_{x} \cdot m_{t}$ unknown coefficients $\left\{u_{i j}\right\}$ are determined by orthogonalizing the residual with respect to the set of sinc functions $\left\{S_{p}(x) S_{q}^{*}(t)\right\}_{p=-M_{0}, \cdots, N_{*}}^{q=-M_{t}, \cdots, N_{t}}$. This yields the discrete Galerkin system

$$
\left(\mathcal{L} u_{m_{u} m_{i}}-f, S_{p} S_{q}^{*}\right)=0
$$

for $p=-M_{x}, \cdots, N_{x}$ and $q=-M_{t}, \cdots, N_{t}$. The inner product $(\cdot, \cdot)$ is taken to be

$$
(F, G)=\int_{0}^{\infty} \int_{0}^{1} F(x, t) G(x, t) w(x, t) d x d t
$$

where

$$
w(x, t)=w(x) w^{*}(t)=\left(\phi^{\prime}(x)\right)^{-\frac{1}{2}}(\dot{\Upsilon}(t))^{-\frac{1}{3}}
$$

The quadrature rules and one-dimensional results from Sections 3.1 and 3.3 can be used to determine the resulting matrix system. 
As before, equating asymptotic errors is fundamental to minimizing system size. When the decay conditions (3.13) or (3.26), and (3.57) are combined to yield

$$
|E I(x) u(x, t)| \leq K x^{\alpha+\frac{1}{2}}(1-x)^{\beta+\frac{1}{3}} t^{\gamma+\frac{1}{2}} e^{-\delta t}
$$

or

$$
|\mathcal{E} \mathcal{I}(x) u(x, t)| \leq K x^{\alpha+\frac{1}{2}}(1-x)^{\beta+\frac{1}{2}} t^{\gamma+\frac{1}{3}} e^{-\delta t}
$$

then the choices

$$
\begin{gathered}
h_{x}=\sqrt{\frac{\pi d}{\alpha M_{x}}}, \\
h_{t}=h_{x}, \\
N_{x}=\llbracket \frac{\alpha}{\beta} M_{x}+1 \rrbracket, \\
M_{t}=\llbracket \frac{\alpha}{\gamma} M_{x}+1 \rrbracket,
\end{gathered}
$$

and

$$
N_{t}=\llbracket \frac{1}{h_{t}} \ln \left(\frac{\gamma}{\delta} M_{t} h_{t}\right)+1 \rrbracket
$$

for the stepsizes and summation limits balance the asymptotic errors. If one takes $d=\frac{\pi}{2}$, then the above choices yield an asymptotic error rate of order $\mathcal{O}\left(e^{-\pi \sqrt{\alpha M_{*} / 2}}\right)$.

Given $M_{x}, N_{x}, M_{t}, N_{t}$ and $h \equiv h_{x}=h_{t}$ as defined above, the discrete system for (4.1) is

$$
A_{x} U C_{t}^{T}+C_{x} U A_{t}^{T}=G
$$

Here

$$
\begin{aligned}
& C_{t} \equiv \mathcal{D}\left(\frac{w^{*}}{\grave{\Upsilon}}\right), \\
& C_{x} \equiv \mathcal{D}\left(\frac{w}{\phi^{\prime}}\right),
\end{aligned}
$$

and

$$
G \equiv \mathcal{D}\left(\frac{w}{\phi^{\prime}}\right) F \mathcal{D}\left(\frac{w^{*}}{\dot{\Upsilon}}\right) .
$$

The $m_{t} \times m_{t}$ matrix $A_{t}$ is given by

$$
A_{t}=\left[\frac{1}{h_{\ell}^{2}} I^{(2)}-\frac{1}{4} I\right] \mathcal{D}\left((\dot{\Upsilon})^{\frac{1}{2}}\right)
$$


as shown in (3.60). Furthermore, $m_{x} \times m_{t}$ matrices $U$ and $F$ are defined componentwise by

$$
[U]_{i j}=u_{i j}
$$

and

$$
[F]_{i j}=f\left(x_{i}, t_{j}\right) .
$$

It should be noted that the ordering of the coefficients $u_{i j}$ in $U$ mimics that used in most standard time-differencing schemes. This is a matter of convenience since the Sinc-Galerkin method is not bound by any specific ordering of the grid.

The structure of the $m_{x} \times m_{x}$ matrix $A_{x}$ depends on the scheme that is used to discretize $E I(x)$. If the parameter is fully differentiated, then $A_{x}$ is given by (3.17). If, on the other hand, $E I(x)$ is approximated by a linear combination of sinc and algebraic basis functions, then $A_{x}$ is given by (3.29).

Various methods exist for solving the equation (4.10). Referred to as a generalized Sylvester equation (4.10) is algebraically equivalent (page 414 of [15]) to the system

$$
\mathcal{A} \vec{u} \equiv\left\{C_{t} \otimes A_{x}+A_{t} \otimes C_{x}\right\} \cot (U)=\operatorname{co}(\vec{G})
$$

where the tensor or Kronecker product of an $m \times m$ matrix $E$ with a $p \times q$ matrix $H$ is defined by

$$
E \otimes H=\left[e_{i j} H\right]_{m p \times n q}
$$

The vector $\vec{u}=c o(\vec{U})$ is the concatenation of the $m_{x} \times m_{t}$ matrix $U$ obtained by successively "stacking" the columns of $U$, one upon another, to obtain an $m_{x} m_{t} \times 1$ vector.

The system (4.14) can be solved directly via any of the decomposition methods that are available for linear systems. Although this system is easily formulated, the fact that $\mathcal{A}$ is very large $\left(m_{x} \cdot m_{t} \times m_{x} \cdot m_{t}\right)$ and not banded causes this method to be impractical in some problems. For more general fourth-order operators however, this may be the only method of choice.

A second algorithm for solving (4.10) depends on the generalized Schur decomposition (page 396 of [16]). As guaranteed by the results of Moler and Stewart [17], there exist unitary 
matrices $Q_{1}, Z_{1}, Q_{2}$, and $Z_{2}$ such that

$$
\begin{aligned}
& Q_{1}^{*} A_{x} Z_{1}=P \\
& Q_{1}^{*} C_{x} Z_{1}=R \\
& Q_{2}^{*} C_{t} Z_{2}=S \\
& Q_{2}^{*} A_{t} Z_{2}=T
\end{aligned}
$$

where $P, R, S$, and $T$ are upper triangular. If $Y=Z_{1}^{*} U Z_{2}$ and $C=Q_{1}^{*} G Q_{2}$, then (4.10) transforms to

$$
P Y T^{*}+R Y S^{*}=C .
$$

By comparing the $k$-th columns, one finds that

$$
P \sum_{j=k}^{n} t_{k j} y_{j}+R \sum_{j=k}^{n} s_{k j} y_{j}=c_{k}
$$

which yields

$$
\left(t_{k k} P+s_{k k} R\right) y_{k}=c_{k}-P \sum_{j=k+1}^{n} t_{k j} y_{j}-R \sum_{j=k+1}^{n} s_{k j} y_{j}
$$

(for convenience, it is assumed that all matrices are $n \times n$ and indexed from 1 to $n$ ). With the assumption that the matrix $\left(t_{k k} P+s_{k k} R\right)$ is nonsingular, the solution to (4.15) is easily found by recursively solving triangular systems.

Although this algorithm does require complex algebra, it is both robust and efficient and requires no assumptions concerning the diagonalizability of the component matrices. It should be noted that a "real" version of this algorithm also exists [18]. In this latter algorithm, $Q_{1}, Z_{1}, Q_{2}$, and $Z_{2}$ are orthogonal with $P, S$ quasi-upper triangular and $R, T$ upper triangular. 


\subsection{The Time-Dependent Problem: Cantilever Boundary Conditions}

A generalization of the problem which arises when modeling beams with cantilever boundary conditions is

$$
\begin{gathered}
\mathcal{L} u(x, t) \equiv \frac{\partial^{2} u}{\partial t^{2}}(x, t)+\frac{\partial^{2}}{\partial x^{2}}\left(E I(x) \frac{\partial^{2} u}{\partial x^{2}}(x, t)\right)=f(x, t), \quad 0<x<1 \\
u(0, t)=\bar{\alpha}(t), \quad\left(E I \frac{\partial^{2} u}{\partial x^{2}}\right)(1, t)=\bar{\gamma}(t), \quad t>0 \\
\frac{\partial u}{\partial x}(0, t)=\bar{\beta}(t), \quad \frac{\partial}{\partial x}\left(E I \frac{\partial^{2} u}{\partial x^{2}}\right)(1, t)=\delta(t), \quad t>0 \\
u(x, 0)=\frac{\partial u}{\partial t}(x, 0)=0, \quad 0 \leq x \leq 1 .
\end{gathered}
$$

The basis for this problem is taken to be $\left\{\zeta_{i}(x) S_{j}^{*}(t)\right\}$ where $\zeta_{i}(x)$ is defined in (3.41) and $S_{j}^{*}(t)=S\left(j, h_{t}\right) \circ \Upsilon(t)$. Here the approximate solution is taken to be

$$
\begin{aligned}
u_{m_{\bullet} m_{t}}(x, t)= & \sum_{i=-M_{\bullet}}^{N_{\bullet}} \sum_{j=-M_{t}}^{N_{t}} u_{i j} \zeta_{i}(x) S_{j}^{*}(t) \\
& +\sum_{j=-M_{t}}^{N_{t}} S_{j}^{*}(t)\left\{u_{-M_{\bullet}-3, j} \zeta_{-M_{\bullet}-3}(x)+u_{-M_{\bullet}-4, j} \zeta_{-M_{\bullet}-4}(x)\right. \\
& \left.+u_{N_{\bullet}+1, j} \zeta_{N_{\bullet}+1}(x)+u_{N_{\bullet}+2, j} \zeta_{N_{\bullet}+2}(x)\right\} \\
& +\left\{\bar{\alpha}(t) \zeta_{-M_{\bullet}-1}(x)+\bar{\beta} \zeta_{-M_{\bullet}-2}(x)+\tilde{\gamma}(t) \zeta_{N_{\bullet}+3}(x)+\tilde{\delta}(t) \zeta_{N_{\bullet}+4}(x)\right\}
\end{aligned}
$$

where

$$
\tilde{\gamma}(t)=\frac{1}{E I(1)} \bar{\gamma}(t)
$$

and

$$
\tilde{\delta}(t)=\frac{1}{E I(1)} \bar{\delta}(t)-\frac{E I^{\prime}(1)}{[E I(1)]^{2}} \bar{\gamma}(t)
$$

It should be noted that the approximate solution does satisfy the boundary conditions in (4.16).

The $\left(m_{x}+4\right) \cdot m_{t}$ unknowns $\left\{u_{i j}\right\}$ are determined by orthogonalizing the residual with respect to the sinc functions $\left\{S_{p}(x) S_{q}^{*}(t)\right\}_{p=-M_{0}-M_{t}, \cdots, N_{\bullet}+2}^{-M_{t}, N_{t}}$. This yields the discrete system

$$
\begin{array}{cl}
\left(\mathcal{L} u_{m_{\bullet} m_{t}}-f, S_{p} S_{q}^{*}\right)=0, & -M_{x}-2 \leq p \leq N_{x}+2 \\
& -M_{t} \leq q \leq N_{t}
\end{array}
$$


where $(\cdot, \cdot)$ is defined in $(4.2)$ with $w(x, t)=(\dot{\Upsilon}(t))^{-\frac{1}{2}}$.

Appropriate integration by parts and the application of the one-dimensional results from Sections 3.2 and 3.3 yields the matrix equation

$$
A_{x} U C_{t}^{T}+C_{x} M U A_{t}^{T}=G
$$

where $C_{t}, C_{x}$ and $A_{t}$ are defined in (4.11), (4.12), and (4.13) respectively, and

$$
G=\mathcal{D}\left(\frac{w}{\phi^{\prime}}\right) F \mathcal{D}\left(\frac{w^{*}}{\dot{\Upsilon}}\right)
$$

The $\left(m_{x}+4\right) \times m_{t}$ matrices $U$ and $\bar{F}$ are defined componentwise by

$$
[U]_{i j}=u_{i j}
$$

and

$$
\bar{F}]_{i j}=\bar{f}\left(x_{i}, t_{j}\right)
$$

where

$$
\begin{aligned}
f(x, t) \equiv & f(x, t)-\mathcal{L}\left(\bar{\alpha}(t) B_{-M_{\bullet}-1}(x)\right)-\mathcal{L}\left(\bar{\beta}(t) B_{-M_{\bullet}-2}(x)\right) \\
& -\mathcal{L}\left(\tilde{\gamma}(t) B_{N_{\bullet}+3}(x)\right)-\mathcal{L}\left(\tilde{\delta}(t) B_{N_{\bullet}+4}(x)\right)
\end{aligned}
$$

The $\left(m_{x}+4\right) \times\left(m_{x}+4\right)$ matrix $A_{x}$ is given by (3.50) or (3.52) depending on which scheme is used to discretize $E I$. Finally the $\left(m_{x}+4\right) \times\left(m_{x}+4\right)$ matrix $M$ has the form

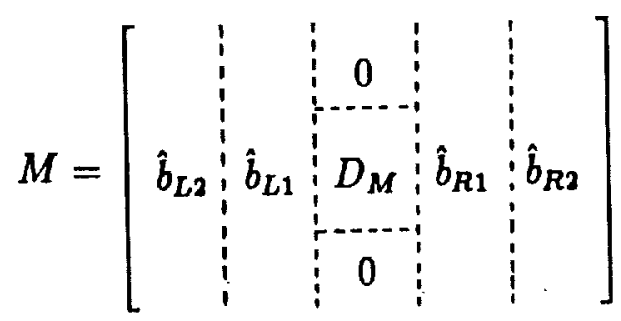

where the $m_{x} \times m_{x}$ submatrix $D_{M}$ and the $\left(m_{x}+4\right) \times 1$ vectors are given by

$$
\begin{gathered}
D_{M}=\mathcal{D}(v), \\
\hat{b}_{L 2}=\mathcal{D}\left(B_{-M_{\bullet-4}}\right) \overrightarrow{1}, \\
\hat{b}_{L 1}=\mathcal{D}\left(B_{-M_{\bullet-3}}\right) \overrightarrow{1}, \\
\hat{b}_{R 1}=\mathcal{D}\left(B_{N_{\bullet}+1}\right) \overrightarrow{1}
\end{gathered}
$$


and

$$
\hat{b}_{R 2}=\mathcal{D}\left(B_{N_{ \pm}+2}\right) \overrightarrow{1}
$$

The system (4.17) can again be solved via the generalized Schur algorithm (4.15) as discussed in Section 4.1 .

Before implementing the method, the decay parameters $\delta, \beta, \gamma$ and $\delta$ must be determined and summation limits chosen. For the spatial weight $w(x)=1$, the decay conditions are

$$
|E I(x) \mathcal{U}(x, t)| \leq x^{\alpha+3}(1-x)^{\beta+3} t^{\gamma+\frac{1}{3}} e^{-\delta t}
$$

or

$$
|\mathcal{E} \mathcal{I}(x) \mathcal{U}(x, t)| \leq x^{\alpha+3}(1-x)^{\beta+3} t^{\gamma+\frac{1}{3}} e^{-\delta t}
$$

depending on the manner in which $E I$ was discretized. Here $\mathcal{E I}(x)$ is defined in (3.27) and $\mathcal{U}(x, t)$ denotes that part of the true solution which is approximated by

$$
u_{h}(x, t)=\sum_{i=-M_{m}}^{N_{k}} \sum_{j=-M_{t}}^{N_{t}} u_{i j} \zeta_{i}(x) S_{j}^{*}(t)
$$

(see also (3.49)). With the decay parameters specified and $M_{x}$ chosen, the remaining stepsizes and summation limits are given by (4.5) - (4.9).

\section{Numerical Examples}

The four examples reported in this section were selected from a large collection of problems to which the Sinc-Galerkin method was applied. The results are representative of those obtained on other sample problems. For purposes of comparison, contrast, and performance evaluation, examples with known solutions were chosen. The first and last examples have analytic solutions, the second example has an algebraic singularity, and the third example contains a logarithmic singularity. As will be demonstrated by the numerical results, the boundary singularities have no adverse affect on the performance of the method. 
In all examples $d=\frac{\pi}{2}$. The errors are reported on both the set of sinc gridpoints

$$
S=\left\{\left(x_{i}, t_{j}\right)\right\}_{i=-M_{\bullet}, \cdots, N_{\bullet} ;}^{j=-M_{t}, \cdots, N_{t}} ; \quad x_{i}=\frac{e^{i h_{\bullet}}}{1+e^{i h_{\star}}}, \quad t_{j}=e^{j h_{t}}
$$

and the set of uniform gridpoints $\left(\ell_{x}=\frac{1}{80}, \ell_{t}=\frac{1}{B}\right)$

$$
U=\left\{\left(z_{p}, s_{q}\right)\right\}_{p=1, \ldots 50}^{q=1, \ldots, 50} ; \quad z_{p}=\ell_{x} p, \quad s_{q}=\ell_{t} q
$$

The errors on these grids are reported as

$$
\left\|E_{S}^{M_{*}}\left(h_{x}, h_{t}\right)\right\|=\max _{\substack{-M_{*} \leq i \leq N_{*} \\-M_{i} \leq j \leq N_{i}}}\left|u\left(x_{i}, t_{j}\right)-u_{m_{e} m_{t}}\left(x_{i}, t_{j}\right)\right|
$$

and

$$
\left\|E_{U}^{M_{*}}\left(\ell_{x}, \ell_{t}\right)\right\|=\max _{\substack{0 \leq p \leq 50 \\ 0 \leq q \leq 50}}\left|u\left(z_{p}, s_{q}\right)-u_{m_{*} m_{t}}\left(z_{p}, s_{q}\right)\right|
$$

respectively. The dependence of both errors on the number of sinc basis functions is indicated with the superscript $M_{x}$. It is noted that if exponential convergence is realized, then

$$
\left(\left\|E_{U}^{M_{*}}\left(\ell_{x}, \ell_{t}\right)\right\|\right)^{\sqrt{\bar{M}_{*} / M_{*}}} \approx\left\|E_{U}^{\tilde{M}_{*}}\left(\ell_{x}, \ell_{t}\right)\right\|
$$

where $M_{x}$ and $\tilde{M}_{x}$ denote the lower limits for the spatial sums. In the examples of this section, $\bar{M}_{x}=2 M_{x}$, and the exponential convergence is verified by comparing $\left(\left\|E_{U}^{\tilde{M}_{*} / 2}\left(\ell_{x}, \ell_{t}\right)\right\|\right)^{\sqrt{2}}$ and $\left\|E_{U}^{\bar{M}_{*}}\left(\ell_{x}, \ell_{t}\right)\right\|$.

The error and convergence results are tabulated in the form .aaa $-\gamma$ which represents $. a a a \times 10^{-\gamma}$. All problems were run with sixteen place accuracy on a Vax 8550 .

Example 5.1.

$$
\begin{gathered}
\frac{\partial^{2} u}{\partial t^{2}}(x, t)+\frac{\partial^{2}}{\partial x^{2}}\left(E I(x) \frac{\partial^{2} u}{\partial x^{2}}(x, t)\right)=f(x, t), \quad 0<x<1 \quad t>0 \\
u(0, t)=u(1, t)=0, \quad t>0 \\
\frac{\partial u}{\partial x}(0, t)=\frac{\partial u}{\partial x}(1, t)=0, \quad t>0 \\
u(x, 0)=\frac{\partial u}{\partial t}(x, 0)=0, \quad 0 \leq x \leq 1
\end{gathered}
$$

The flexural rigidity is $E I(x)=1+\sin (\pi x)$ and $f(x, t)$ is consistent with the true solution $u(x, t)=x(1-x) \sin (4 \pi x) t^{2} e^{-t}$. The parameter is expanded via (3.25) thus yielding the 
spatial representation (3.29). The decay condition (4.4) dictates the parameter choices $\alpha=$ $\beta=\gamma=\frac{3}{2}$ and $\delta=1$. The asymptotic error rate $\mathcal{O}\left(e^{-\pi \sqrt{3 M_{*} / 2}}\right)$ is maintained as indicated by the last column of Table 1 . Notice that the choice of $N_{t}$ given in (4.9) significantly reduces the size of the matrices involved in (4.10). The errors on both the sinc grid and the uniform grid do not differ dramatically though in the next example the difference will be more noticeable. Figure 3 shows the true solution $u(x, t)$ while Figure 4 shows both the true and approximate solutions (for $M_{x}=8,16$ ) at the time slice $t=2$. The approximate solution for $M_{x}=32$ is buried in the true solution on this scale.

\begin{tabular}{ccccccc}
\hline$M_{x}$ & $N_{x}$ & $M_{t}$ & $N_{t}$ & $\left\|E_{S}^{M_{*}}\left(h_{x}, h_{t}\right)\right\|$ & $\left\|E_{U}^{M_{*}}\left(\ell_{x}, \ell_{t}\right)\right\|$ & $\left(\left\|E_{U}^{M_{*} / 2}\left(\ell_{x}, \ell_{t}\right)\right\|\right)^{\sqrt{2}}$ \\
\hline 4 & 4 & 4 & 2 & $.6207-0$ & $.8040-0$ & - \\
8 & 8 & 8 & 4 & $.6890-1$ & $.8448-1$ & $.7345-0$ \\
16 & 16 & 16 & 6 & $.2910-3$ & $.1105-2$ & $.3035-1$ \\
32 & 32 & 32 & 9 & $.1906-4$ & $.2151-4$ & $.6587-4$ \\
\hline
\end{tabular}

Table 1. Errors on the Sinc Grid $S$ and the Uniform Grid $U$ for Example 5.1. 


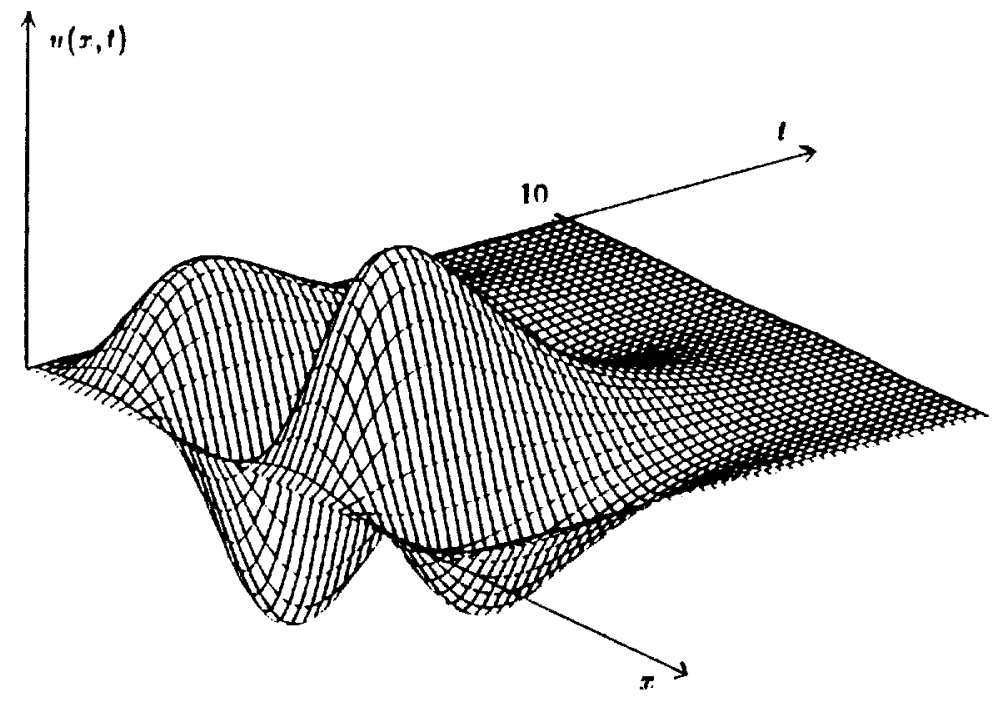

Figure 3. True Solution to Example 5.1.

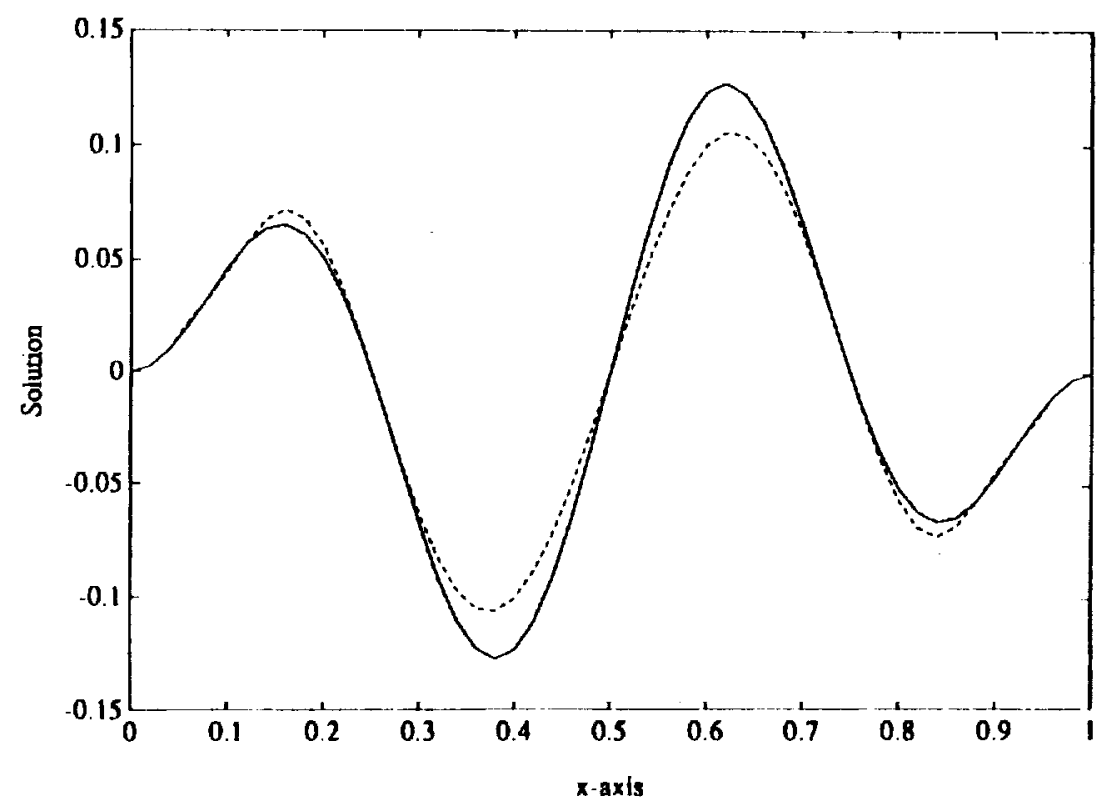

Figure 4. True and Approximate Solutions to Example 5.1 at Time $t=2$

$$
---\left(M_{x}=8\right),-\cdot-\left(M_{x}=16\right),- \text { (True). }
$$


Example 5.2.

$$
\begin{gathered}
\frac{\partial^{2} u}{\partial t^{2}}(x, t)+\frac{\partial^{4} u}{\partial x^{4}}(x, t)=f(x, t), \quad 0<x<1 \quad t>0 \\
u(0, t)=u(1, t)=0, \quad t>0 \\
\frac{\partial u}{\partial x}(0, t)=\frac{\partial u}{\partial x}(1, t)=0, \quad t>0 \\
u(x, 0)=\frac{\partial u}{\partial t}(x, 0)=0, \quad 0 \leq x \leq 1
\end{gathered}
$$

The function $f(x, t)$ is consistent with the solution $u(x, t)=[x(1-x)]^{7 / 2} t^{5 / 2} e^{-t}$ which has algebraic singularities at $x=0, x=1$ and $t=0$. The spatial discretization is taken to be (3.17) with the decay parameters $\alpha=\beta=\gamma=2, \delta=1$ dictated by (4.3). As indicated by Table 2, the asymptotic error rate $O\left(e^{-\pi \sqrt{M_{*}}}\right)$ is achieved in spite of the boundary singularities. The increased accuracy of the method for this problem as compared to Example 5.1 is due to the larger values of $\alpha, \beta$ and $\gamma$. Here the error on the sinc grid is substantially smaller than that on the uniform grid. This emphasizes that one should use caution when assessing performance of a method based on only the errors at the gridpoints of the method. The true solution is plotted in Figure 5 while time slices (at time $t=2$ ) of both the true and approximate solutions (for $M_{x}=4,8$ ) are plotted in Figure 6.

\begin{tabular}{ccccccc}
\hline$M_{x}$ & $N_{x}$ & $M_{t}$ & $N_{t}$ & $\left\|E_{S}^{M_{*}}\left(h_{x}, h_{t}\right)\right\|$ & $\left\|E_{U}^{M_{*}}\left(\ell_{x}, \ell_{t}\right)\right\|$ & $\left(\left\|E_{U}^{M_{*} / 2}\left(\ell_{x}, \ell_{t}\right)\right\|\right)^{\sqrt{2}}$ \\
\hline 4 & 4 & 4 & 3 & $.2040-4$ & $.3184-3$ & - \\
8 & 8 & 8 & 4 & $.9361-5$ & $.3618-4$ & $.1134-4$ \\
16 & 16 & 16 & 7 & $.1372-6$ & $.1788-5$ & $.5233-6$ \\
32 & 32 & 32 & 11 & $.1742-9$ & $.1338-7$ & $.7441-8$ \\
\hline
\end{tabular}

Table 2. Errors on the Sinc Grid $S$ and the Uniform Grid $U$ for Example 5.2. 


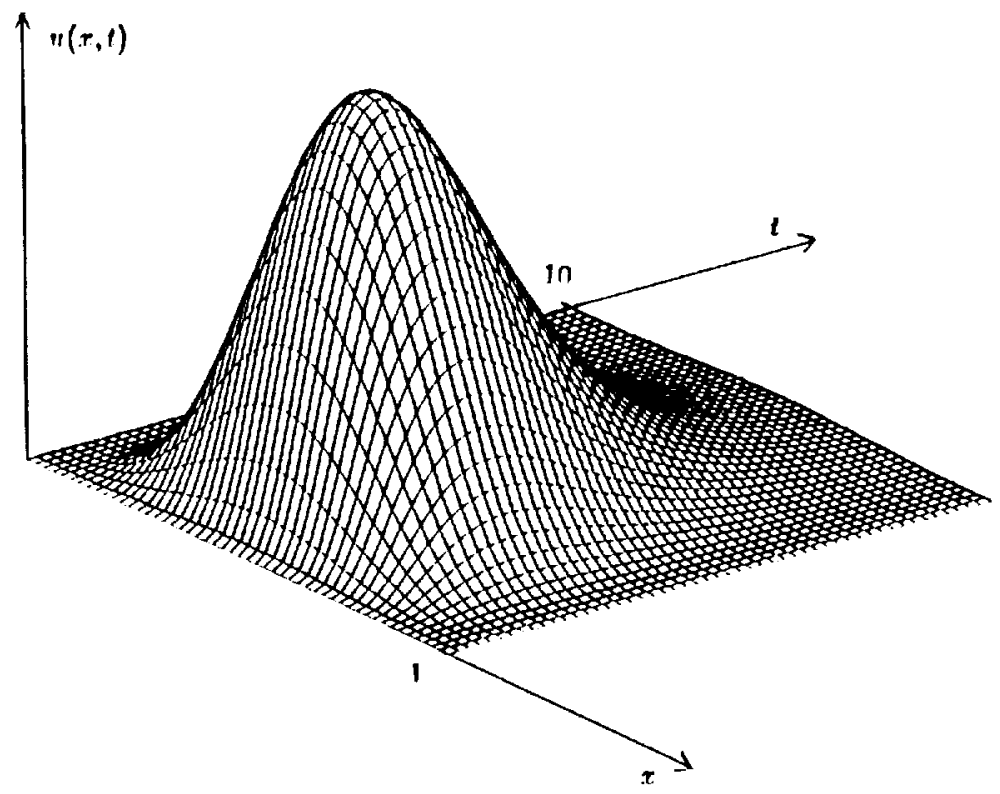

Figure 5. True Solution to Example 5.2.

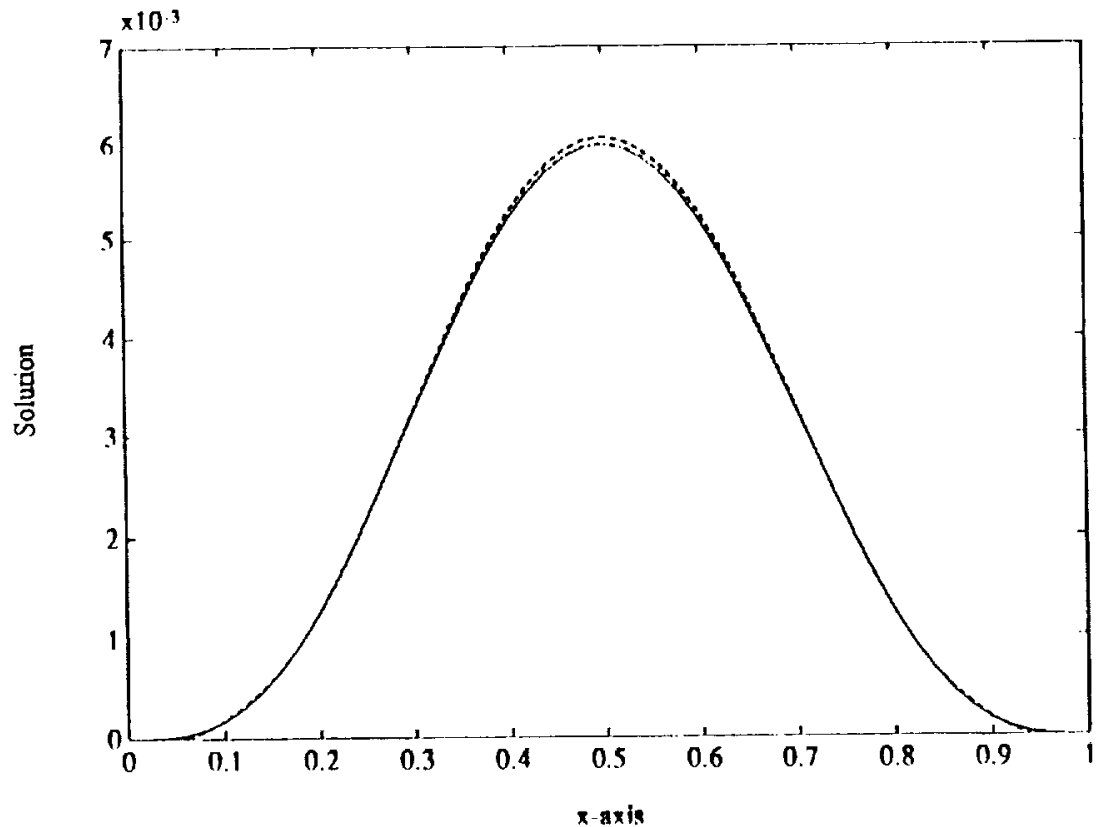

Figure 6. True and Approximate Solutions to Example 5.2 at Time $t=2$

$$
---\left(M_{x}=4\right),-\cdots\left(M_{x}=8\right), \longrightarrow \text { (True). }
$$


Example 5.9.

$$
\begin{gathered}
\frac{\partial^{2} u}{\partial t^{2}}(x, t)+\frac{\partial^{4} u}{\partial x^{4}}(x, t)=f(x, t), \quad 0<x<1 \quad t>0 \\
u(0, t)=0, \quad \frac{\partial^{2} u}{\partial x^{2}}(1, t)=2 t^{2} e^{-t}, \quad t>0 \\
\frac{\partial u}{\partial x}(0, t)=0, \quad \frac{\partial^{3} u}{\partial x^{3}}(1, t)=0, \quad t>0 \\
u(x, 0)=\frac{\partial u}{\partial t}(x, 0)=0, \quad 0 \leq x \leq 1 .
\end{gathered}
$$

The true solution $u(x, t)=\left[(x \ln (x))^{4}+x^{2}\right] t^{2} e^{-t}$ dictates the forcing function $f(x, t)$. The decay condition (4.18) with $E I(x)=1$ yields the parameter choices $\alpha=\beta=1, \gamma=\frac{3}{2}$, and $\delta=1$ which in turn implies the asymptotic error rate $\mathcal{O}\left(e^{-\pi \sqrt{M_{*} / 2}}\right)$. As indicated by the results in Table 3 this rate is achieved in spite of the logarithmic singularity at $x=0$. The convergence of the method is even accelerated which can be seen by in the last column of Table 3. The mesh plot in Figure 7 shows the distinctive behavior that the solution can exhibit when cantilever boundary conditions are in force. The "oscillation" at the right-hand end is tracked accurately by this method. The time slice at $t=2$ shown in Figure 8 shows the approximate $\left(M_{x}=4,8,16\right)$ as well as the true solution.

\begin{tabular}{ccccccc}
\hline$M_{x}$ & $N_{x}$ & $M_{t}$ & $N_{t}$ & $\left\|E_{S}^{M_{*}}\left(h_{x}, h_{t}\right)\right\| \cdot\left\|E_{U}^{M_{*}}\left(\ell_{x}, \ell_{t}\right)\right\|$ & $\left(\left\|E_{U}^{M_{*} / 2}\left(\ell_{x}, \ell_{t}\right)\right\|\right)^{\sqrt{2}}$ \\
\hline 4 & 4 & 3 & 2 & $.4227-1$ & $.9083-1$ & - \\
8 & 8 & 6 & 3 & $.8034-2$ & $.1999-1$ & $.3363-1$ \\
16 & 16 & 11 & 4 & $.8799-3$ & $.1838-2$ & $.3953-2$ \\
32 & 32 & 22 & 7 & $.3947-4$ & $.8741-4$ & $.1353-2$ \\
\hline
\end{tabular}

Table 3. Errors on the Sinc Grid $S$ and the Uniform Grid $U$ for Example 5.3. 


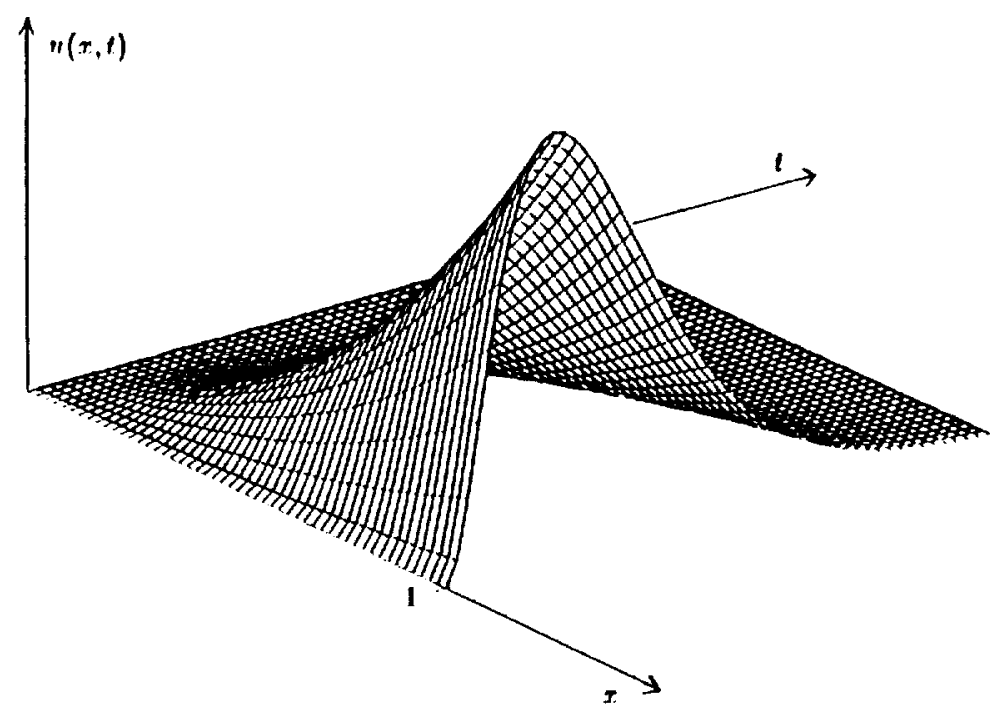

Figure 7. True Solution to Example 5.3.

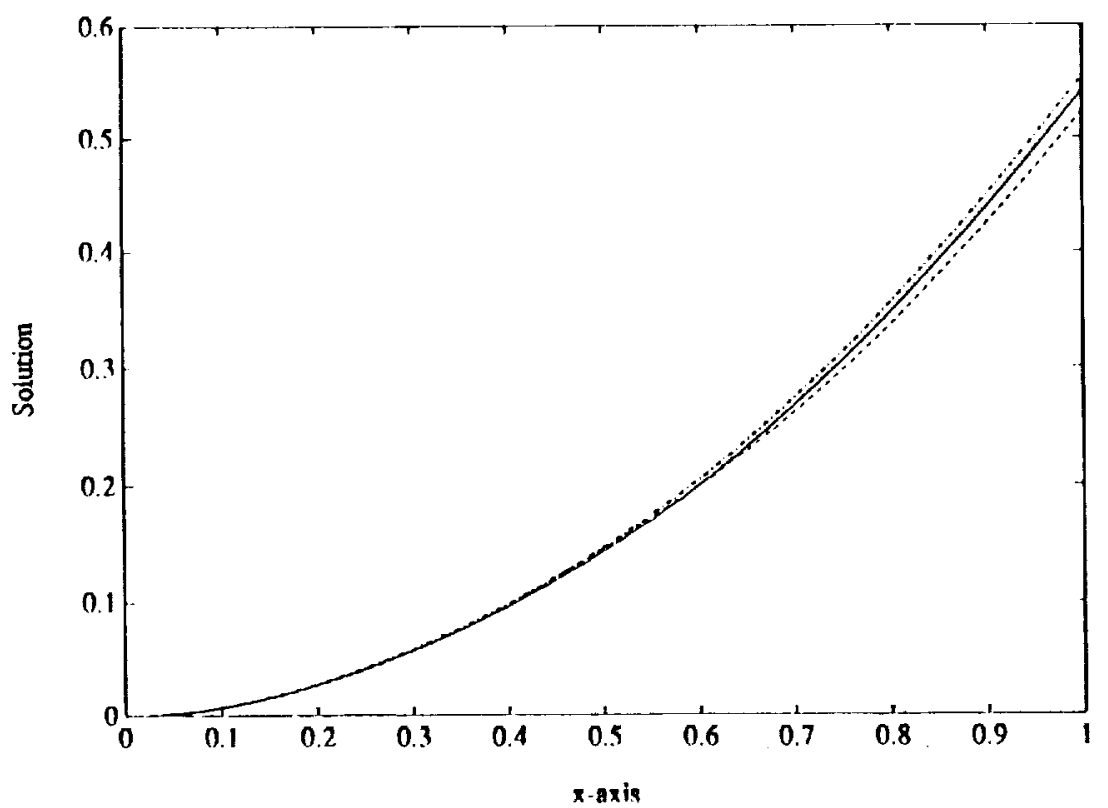

Figure 8. True and Approximate Solutions to Example 5.3 at Time $t=2$

$$
---\left(M_{x}=4\right),-\cdot-\left(M_{x}=8\right), \cdots\left(M_{x}=16\right),- \text { (True). }
$$


Example 5.4.

$$
\begin{gathered}
\frac{\partial^{2} u}{\partial t^{2}}(x, t)+\frac{\partial^{2}}{\partial x^{2}}\left(E I(x) \frac{\partial^{2} u}{\partial x^{2}}(x, t)\right)=f(x, t), \quad 0<x<1 \quad t>0 \\
u(0, t)=0, \quad\left(E I \frac{\partial^{2} u}{\partial x^{2}}\right)(1, t)=0, \quad t>0 \\
\frac{\partial u}{\partial x}(0, t)=t^{2} e^{-t}, \quad \frac{\partial}{\partial x}\left(E I \frac{\partial^{2} u}{\partial x^{2}}\right)(1, t)=8 \pi^{3} t^{2} e^{-t}, \quad t>0 \\
u(x, 0)=\frac{\partial u}{\partial t}(x, 0)=0, \quad 0 \leq x \leq 1
\end{gathered}
$$

This example illustrates a problem where the spatial discretization (3.52) is useful. Here the flexural rigidity is $E I(x)=1+\sin (\pi x)$ and the forcing function $f(x, t)$ is consistent with the true solution $u(x, t)=\left[\sin ^{3}(\pi x)+x\right] t^{2} e^{-t}$. The parameter $E I(x)$ is expanded via (3.25) thus yielding the spatial representation (3.52). The parameter choices $\alpha=\beta=1$, $\gamma=\frac{3}{2}$ and $\delta=1$ follow from (4.19). As demonstrated by the last column of Table 4, the asymptotic error rate $\mathcal{O}\left(e^{-\pi \sqrt{M_{*} / 2}}\right)$ is achieved for larger values of $M_{x}, N_{x}, M_{t}$, and $N_{t}$. On this example the errors on the sinc grid and those on the uniform grid are nearly the same. The smaller parameters $\alpha$ and $\beta$ indicate why the errors here are larger than in the previous three examples. A mesh plot of the true solution is shown in Figure 9 while a time slice $(t=2)$ of both the true and approximate solutions $\left(M_{x}=8,16\right)$ are plotted in Figure 10.

\begin{tabular}{ccccccc}
\hline$M_{x}$ & $N_{x}$ & $M_{t}$ & $N_{t}$ & $\left\|E_{S}^{M_{*}}\left(h_{x}, h_{t}\right)\right\|$ & $\left\|E_{U}^{M_{*}}\left(\ell_{x}, \ell_{t}\right)\right\|$ & $\left(\left\|E_{U}^{M_{*} / 2}\left(\ell_{x}, \ell_{t}\right)\right\|\right)^{\sqrt{2}}$ \\
\hline 8 & 8 & 6 & 3 & $.6889-1$ & $.6958-1$ & - \\
16 & 16 & 11 & 4 & $.2958-1$ & $.3165-1$ & $.2307-1$ \\
32 & 32 & 22 & 7 & $.4346-3$ & $.4885-3$ & $.7572-2$ \\
\hline
\end{tabular}

Table 4. Errors on the Sinc Grid $S$ and the Uniform Grid $U$ for Example 5.4. 


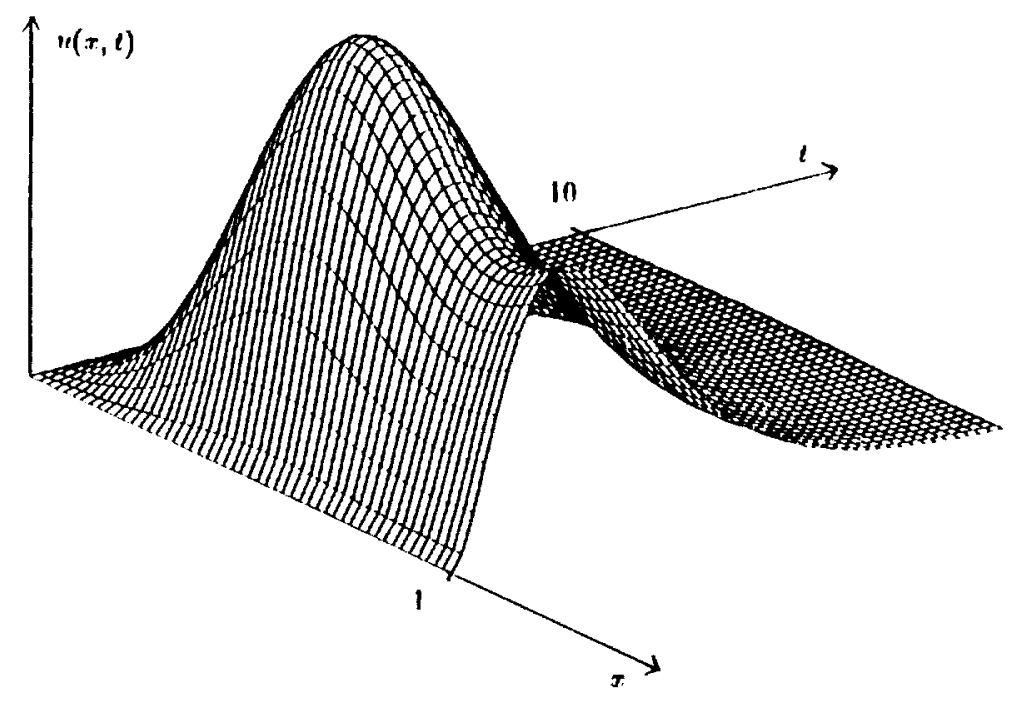

Figure 9. True Solution to Example 5.4.

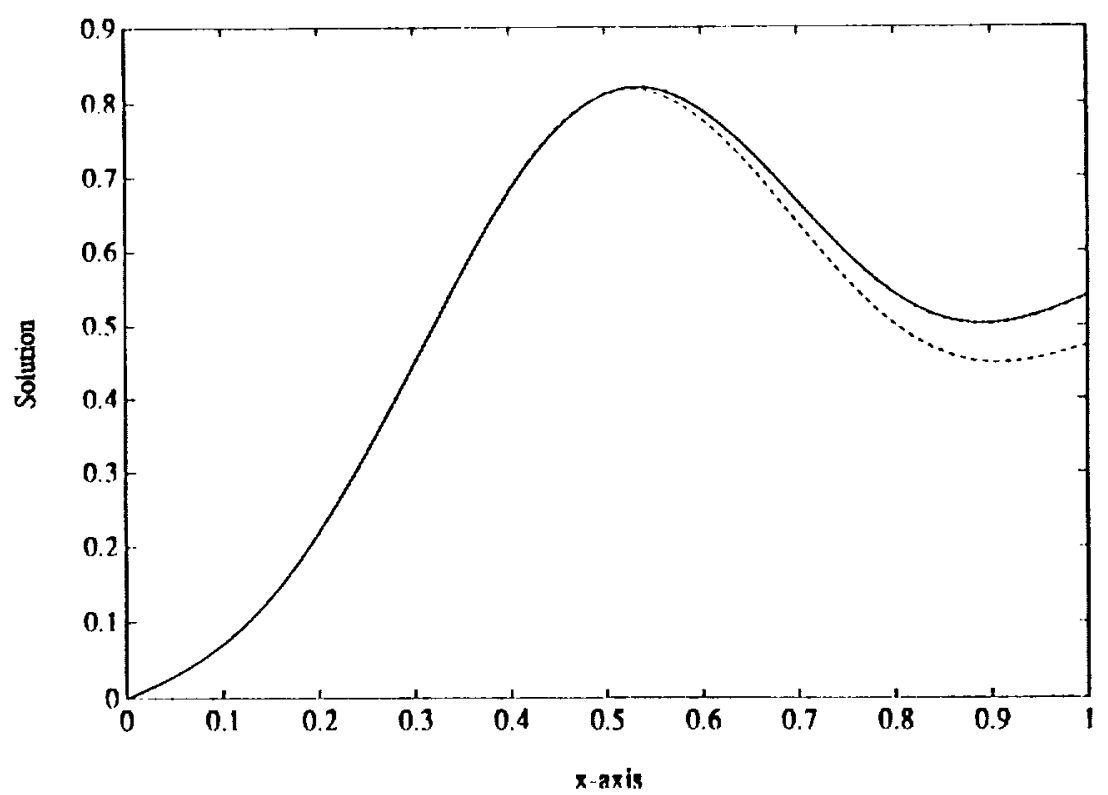

Figure 10. True and Approximate Solutions to Example 5.4 at Time $t=2$

$$
--\left(M_{x}=8,\right)-\cdots\left(M_{x}=16\right), \longrightarrow \text { (True). }
$$




\section{Conclusions}

A fully Sinc-Galerkin method in both space and time is presented for fourth-order timedependent problems with fixed and cantilever boundary conditions. The sinc basis properties which facilitate the simple assembly of the discrete system are discussed in Section 2. In Section 3 , the sinc discretizations for the second-order temporal problem and the fourth-order spatial problems are presented. Alternate formulations for the variable parameter fourthorder problems are given which prove to be especially useful when applying the forward techniques of this paper to parameter recovery problems. The ODE results are then combined in Section 4 to form the discrete systems corresponding to the time-dependent problems of interest. Computational issues are discussed and a robust and efficient algorithm for solving the resulting matrix system is outlined. Numerical examples which highlight the method are given in Section 5. As demonstrated by the numerical results, the exponential convergence rate of the method is maintained for problems with both analytic and singular solutions as well as fixed and cantilever boundary conditions. 


\section{References}

[1] K.L. Bowers and J. Lund, "Numerical Solution of Singular Poisson Problems Via the Sinc-Galerkin Method," SIAM J. Numer. Anal, 24, 36-51 (1987).

[2] K.M. McArthur, K.L. Bowers and J. Lund, "The Sinc Method in Multiple Space Dimensions: Model Problems," Numer. Math., 56, 789-816 (1990).

[3] D.L. Lewis, J. Lund and K.L. Bowers "The Space-Time Sinc-Galerkin Method for Parabolic Problems," Internat. J. Numer. Methods Engrg., 24, 1629-1644 (1987).

[4] K.M. McArthur, K.L. Bowers and J. Lund, "Numerical Implementation of the SincGalerkin Method for Second-Order Hyperbolic Equations," Numer. Methods Partial Differential Equations, 3, 169-185 (1987).

[5] H.T. Banks and K.Kunisch, Estimation Techniques for Distributed Parameter Systems, Birkhäuser, Boston, 1989.

[6] K. Kunisch and E. Graif, "Parameter Estimation for the Euler-Bernoulli Beam," Mat. Aplicada e Computational, 4, 95-124 (1985).

[7] S.P. Timoshenko and J.M. Gere, Theory of Elastic Stability, McGraw-Hill, New York, 1961:

[8] R.C. Smith, "Numerical Solution of Fourth-Order Time-Dependent Problems with Applications to Parameter Identification," Ph.D. Thesis, Montana State University, 1990.

[9] F. Stenger, "A Sinc-Galerkin Method of Solution of Boundary Value Problems," Math. Comp., 33, 85-109 (1979).

[10] F. Stenger, "Numerical Methods Based on Whittaker Cardinal, or Sinc Functions," SIAM Rev., 23, 165-224 (1981).

[11] F. Stenger, "Approximations Via Whittaker's Cardinal Function," J. Approx. Theory, 17, 222-240 (1976). 
[12] J. Lund, "Symmetrization of the Sinc-Galerkin Method for Boundary Value Problems," Math. Comp., 47, 571-588 (1986).

[13] R.C. Smith, G.A. Bogar, K.L. Bowers and J. Lund, "The Sinc-Galerkin Method for Fourth-Order Differential Equations," to appear in SIAM J. Numer. Anal..

[14] K.M. McArthur, R.C. Smith, K.L. Bowers and J. Lund, "Treatment of Variable Coefficients and Boundary Conditions in Sinc-Galerkin Methods". To be submitted to J. of Computational and Appl. Math.

[15] P. Lancaster and M. Tismenetsky, The Theory of Matrices, Academic Press, Orlando, FL, 1985.

[16] G.H. Golub and C. VanLoan, Matrix Computations, 2nd ed., Johns Hopkins University Press, Baltimore, 1989.

[17] C.B. Moler and G.W. Stewart, "An Algorithm for Generalized Matrix Eigenvalue Problems," SIAM J. Numer. Anal., 10, 241-256 (1973).

[18] G.H. Golub, S. Nash, and C. VanLoan, "A Hessenberg-Schur Method for the Problem $A X+X B=C$," IEEE Trans. Automat. Control, 24, 909-913 (1979). 


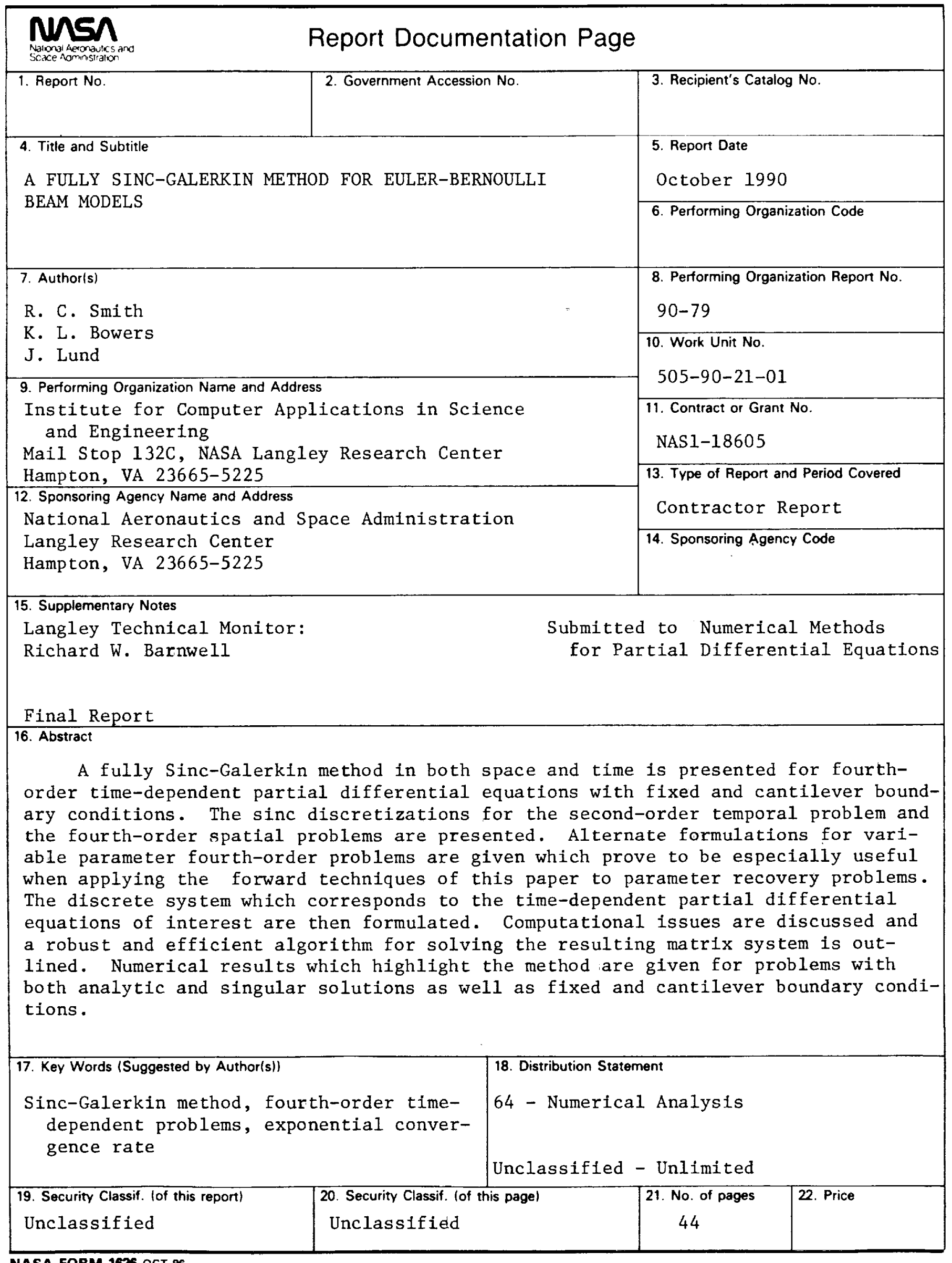


• 